\title{
Revisiting Fuzzy and Linguistic Decision-Making: Scenarios and Challenges for Making Wiser Decisions in a Better Way
}

\author{
Enrique Herrera-Viedma, Senior Member, IEEE; Iván Palomares; Cong-Cong Li; Francisco Javier Cabrerizo; \\ Yucheng Dong, Senior Member, IEEE; Francisco Chiclana; Francisco Herrera, Senior Member, IEEE
}

\begin{abstract}
This paper provides a brief tour through the main fuzzy and linguistic decision-making trends, studies, methodologies and models developed in the last 50 years. Fuzzy and linguistic decision-making approaches allow to address complex real-world decision problems where humans exhibit vagueness, imprecision and/or use natural language to assess decision alternatives, criteria, etc. The aim of this paper is threefold. Firstly, the main fuzzy set theory and computing with words based representation paradigms of decision information, with their different levels of expressive richness and complexity, are reviewed. Secondly, three core decision-making frameworks are examined: (1) multi-criteria decision-making, (2) group consensus-driven decision-making, and (3) multi-person multi-criteria decisionmaking. Thirdly, the paper discusses new complex decisionmaking frameworks that have emerged in recent years, where decisions are guided by the "wisdom of the crowd": their associated challenges are highlighted and considerations on much needed key guidelines for future research in the field are provided.
\end{abstract}

Index Terms-fuzzy decision-making; fuzzy sets; linguistic decision-making; preference modelling; multi-criteria decisionmaking; group decision-making; consensus reaching processes

\section{INTRODUCTION}

Decision-making is a humankind cognitive process consisting in selecting an alternative amid multiple options. Decisionmaking environments can be discriminated with respect to the type of alternative knowledge/assessment on the alternatives to process or analyse: in a certainty environment the knowledge/assessment on the alternatives is clearly known; in a risk environment the knowledge/assessment on the alternatives is probabilistic; while in an uncertainty environment the knowledge/assessment on the alternatives are evaluated in an approximate fashion that is non-probabilistic. Within this last type of environments, fuzzy decision-making, i.e. decisionmaking where the information about the alternatives is vague and imprecise, became a prolific research area after Zadeh's

E. Herrera-Viedma, I. Palomares, F. J. Cabrerizo and F. Herrera are with Andalusian Research Institute of Data Science and Computational Intelligence (DaSCI), University of Granada, Granada, 18071, Spain (email: viedma@decsai.ugr.es, ivanpc@ugr.es, cabrerizo@decsai.ugr.es, herrera@decsai.ugr.es).

F. Chiclana is with Institute of Artificial Intelligence, School of Computer Science and Informatics, De Montfort University, Leicester, UK, and Andalusian Research Institute in Data Science and Computational Intelligence, University of Granada, Granada, Spain (email: chiclana@dmu.ac.uk).

C.-C. $\mathrm{Li}$ is with School of Economics and Management, Southwest Jiaotong University, Chengdu, 610031, China (email: congcongli@ @wjtu.edu.cn).

Y. C. Dong is with Center for Network Big Data and Decision-Making, Business School, Sichuan University, Chengdu 610065, China (e-mail: ycdong@scu.edu.cn). seminal fuzzy sets article in 1965 [1], which was key in the development of the pioneering fuzzy decision-making model by Bellman and Zadeh, published 50 years ago, in 1970 [2].

Since then, fuzzy decision-making scenarios, approaches and methodologies have gained enormous popularity due to their ability to capture human beings' inherent subjectivity and vagueness when facing decision problems of diverse complexity levels. Contributions in this field include models and methods for group and/or multi-criteria decisions [3], [4], [5], fuzzy and linguistic decision-making foundations [6], [7] such as the computing with words paradigm [8], consensual decision approaches [9], fuzzy and linguistic preference modelling [8], [10], [11], aggregation operators [12], [13], and approaches for social network contexts [14], [15], largescale decisions [16], or decisions guided by opinions in social networks [17], amongst others.

This paper aims at paying tribute to research progress made in the fuzzy and linguistic decision-making landscape over the last five decades. In a broad sense, a main objective of this study is to provide an overview of the current state of affairs and potential future research opportunities on this field. To do so, a discussion is set forth on the research challenges regarding (i) making wiser decisions in a better way, (ii) representing expert assessments using realistic and common sense approaches, (iii) incentivising more practical and impactdriven developments, and ultimately (iv) stimulating future developments towards "crowd decision-making" phenomena, i.e. events guided by the wisdom and the intelligence of crowds and aided by recent socio-technological developments.

The rest of the paper is structured as follows. Section II introduces existent approaches proposed to represent assessment information in decision-making based on fuzzy sets, their best known extensions and computing with words, followed by a critical perspective on recent complex approaches far from common sense. Section III examines the characteristics and role of fuzzy and linguistic representation approaches in three core decision-making frameworks with multiple criteria and/or group of people. Section IV explores complex and emerging decision-making paradigms that have attracted recent research interest; highlights their challenges and provides a reflection about prospects and opportunities raised by data, AI and emerging technologies; as well as the imperative paradigm shifts to be adopted for advancing the state of affairs on this research field in future. Finally, Section V presents our concluding remarks. 


\section{INFORMATION REPRESENTATION FORMATS IN UNCERTAIN ENVIRONMENTS}

In decision-making processes, the most suitable alternative is determined among a set of possible options by establishing a ranking of them. This is typically done predicated on knowledge in the form of human expert assessments on the alternatives, which can represent (i) the degree up to which an alternative satisfies a particular criterion or (ii) the preference degree of one alternative over another one. In both cases, the assessment must be provided in a given representation domain. Therefore, a key issue in decision-making processes is the selection of a representation domain to formally represent the assessments expressed by the experts.

Since the process of decision-making is a cognitive process carried out by humans (experts), fuzzy set theory is particularly suitable to be used in its resolution and, since Zadeh introduced it in [1], remarkable progress has been made in this research area with the development of fuzzy set based models of representation of decision information pervaded with human uncertainty regarding their assessment of the alternatives. Indeed, both fuzzy set theory and its extensions [18] are very helpful in dealing with vagueness or ambiguity of human assessments both quantitatively and qualitatively, being the most appropriate tools to characterize often not welldefined assessments found in most practical situations. As a consequence, a number of models and tools based on fuzzy sets and their extensions have been proposed for their use in decision-making processes. In the following subsections, we recall some of the most important ones.

\section{A. Fuzzy number assessments}

The concept of fuzzy set addresses the modelling of belonging of an object in a particular class such as the satisfaction of a particular property which is defined in vague terms, and therefore there is non-probablistic uncertainty regarding the class boundary.

Definition 1: [1] "Let $X$ be a non-empty universal set. A fuzzy set $A$, also called type-1 fuzzy set, on $X$ is defined by a membership function $\mu_{A}: X \rightarrow[0,1]$ that associates each element of the universal set $X, x \in X$, a degree of membership to $A, \mu_{A}(x): A=\left\{\left(x, \mu_{A}(x)\right) \mid x \in X \wedge\right.$ $\left.\mu_{A}(x) \in[0,1]\right\} . "$

Based on the concept of fuzzy set, fuzzy numbers were introduced to manage imprecise numerical quantities in a practical way [19]. They are generalizations of real numbers in the sense that they do not refer to a single value but rather to a connected set of possible values, which have their own weight between 0 and 1 .

Definition 2: [19] "Let $A: \mathbb{R} \rightarrow[0,1]$ be a fuzzy set of the real line. Then, $A$ is a fuzzy number if it satisfies the following properties:

- $A$ is normal, i.e., $\exists x_{0} \in \mathbb{R}$ with $A\left(x_{0}\right)=1$.

- $A$ is fuzzy convex, i.e., $A(t x+(1-t) y) \geq$ $\min \{A(x), A(y)\}, \forall t \in[0,1], x, y \in \mathbb{R}$.

- $A$ is upper semicontinuous on $\mathbb{R}$, i.e., $\forall \epsilon>0 \exists \delta>0$ such that $A(x)-A\left(x_{0}\right)<\epsilon,\left|x-x_{0}\right|<\delta$.
- $A$ is compactly supported, i.e., $\operatorname{cl}\{x \in \mathbb{R} ; A(x)>0\}$ is compact, where $\operatorname{cl}(B)$ denotes the closure of the set $B$."

When using fuzzy numbers, instead of providing an exact value, the expert uses expressions like "about 0.6 " to indicate a real number in a vague but practical way [20].

\section{B. $[0,1]$-assessments}

As per the definition of fuzzy set, the membership degree $\mu_{A}(x)$ has been also used to characterize the experts' assessments. For example, it can represent the degree up to which a certain alternative $x$ satisfies a given criterion $A$ according to the expert's opinion. In addition, a more flexible approach to model experts' uncertain assessment on alternatives consists in the use of interval valued numbers in Definition 1 rather than precise membership degrees, i.e. in considering $\mu_{A}(x)$ to be a closed interval of the unit interval $[0,1][21]$, which are called interval-valued fuzzy set (IVFS).

\section{Atanassov intuitionistic fuzzy assessments}

Zadeh's fuzzy set theory includes the assumption of the complement of a fuzzy set $A$ having membership function uniquely defined in terms of the membership function $\mu_{A}(x)$ as $1-\mu_{A}(x)$. In other words, in Zadeh's fuzzy set theory the addition of the membership degree of each element of the universal set $X$ to a fuzzy set $A$ or its complement $A^{c}$ is equal to 1. This fundamental assumption is relaxed in Atanassov's intuitionistic fuzzy set theory [22], and the mentioned addition is subject to the constraint of being less than or equal to 1. Thus, in Atanassov's intuitionistic fuzzy set theory, the membership degree of each element of the universal set $X$ to the complement fuzzy set of $A$ is no greater than $1-\mu_{A}(x)$. This difference allows to better model hesitation situations with regards to both the exclusion and the inclusion of an object to a particular class [13], [23].

Definition 3: [22] "Let $X$ be a non-empty universe. An Atanassov intuitionistic fuzzy set (AIFS) $A$ on $X$ is defined as $A=\left\{\left(x, \mu_{A}(x), \nu_{A}(x)\right) \mid x \in X\right\}$ with $\mu_{A}(x) \in[0,1]$ being the membership degree of the element $x \in X$ in $A$ and $\nu_{A}(x) \in[0,1]$ being the non-membership degree of the element $x \in X$ in $A$, subject to the constraint $0 \leq \mu_{A}(x)+$ $\nu_{A}(x) \leq 1$."

Atanassov defined the concept of hesitancy index associated with each alternative $x \in X$ in the considered set $A$ as $\pi_{A}(x)=1-\mu_{A}(x)-\nu_{A}(x)$, which represents a measure of the hesitation of the expert to assign a numerical value to $\mu_{A}(x)$ and $\nu_{A}(x)$. The advantage of considering the hesitation index, i.e., another degree of freedom, is that it allows to represent hesitancy of experts [24]. It is noticed here, that AIFSs and IVFSs are mathematically equivalent [25].

\section{Hesitant fuzzy assessments}

Motivated by the natural difficulty often appearing when the membership degree of an element must be established not because of an error margin as in Atanassov intuitionistic fuzzy sets but rather due to having several among which one would be the right one- Torra in [26] introduced a new 
extension of fuzzy sets, called hesitant fuzzy sets, by proposing to represent membership degrees as subsets of $[0,1]$. This situation is very common in decision-making processes when an expert could take into account several membership degrees, i.e. $\{0.54,0.56,0.59\}$ of an object to a particular class [27].

Definition 4: [26] "Let $X$ be a non-empty universe. A hesitant fuzzy set on $X$ is a function $h$ returning a subset of values in $[0,1]$, i.e., $h: X \rightarrow\{[0,1]\}$."

Therefore, a hesitant fuzzy set may also be built from a set of fuzzy sets.

Definition 5: [26] "Let $M=\left\{\mu_{1}, \mu_{2}, \ldots, \mu_{n}\right\}$ be a set of membership functions. The hesitant fuzzy set related to $M, h_{M}$, is defined as $h_{M}: M \rightarrow\{[0,1]\}$ and $h_{M}(x)=$ $\bigcup_{\mu \in M}\{\mu(x)\}$, where $x \in X$."

Note this definition is quite appropriate to decision-making when experts must assess a set of alternatives. Here, $M$ characterizes the assessments of the experts for each alternative and $h_{M}$ the assessments of the group of experts [28].

\section{E. Linguistic assessments}

In view of the fact that natural language is the standard representation of the concepts used by humans for communication, in decision-making processes it has been considered that a promising approach is one where experts provide their assessments via words rather than real numbers (from $[0,1]$ ). The use of natural language implies the need for computing with words (CW) [8], [29], a methodology in which the main computation elements, instead of being numbers, are linguistic variables whose values are propositions and words drawn from a natural language [30], [31], [32].

Definition 6: [30] "A linguistic variable is formally defined as a quintuple $(L, T(L), U, G, M)$ where:

- $L$ is the name of the variable.

- $L(T)$ is the collection of linguistic values of $L$, i.e., the term set of $L$.

- $U$ is a universe of discourse.

- $G$ is the syntactic rule generating the terms in $T(L)$.

- $M$ is the semantic rule associating with each linguistic value $X$ its meaning $M(X)$, where $M(X)$ denotes a fuzzy subset of $U$."

To handle linguistic variables, the linguistic descriptors for the term set and their associated semantics have to be determined. Different approaches to do this has been developed, which may be classified into two groups [33].

1) Approaches based on fuzzy numbers: These approaches, also called semantic approaches, work on fuzzy numbers related to the semantics and employ the extension principle for computation [30]. Within the scope of the semantic approaches, we find (i) approaches based on membership functions [34], and (ii) approaches based on type-2 fuzzy sets [35], concretely, interval type-2 fuzzy sets and interval-valued fuzzy sets (particular cases of type- 2 fuzzy sets) as they keep the uncertainty modelling properties of general type-2 fuzzy sets but decrease the computation efforts required to make computations with them. The drawback of these approaches is that they need an approximation process that leads to a lack of accuracy in the results and a loss of information [29] (critical aspects to be considered in decision-making processes).
2) Approaches based on qualitative scales: These approaches present higher interpretability than the semantic ones and simpler computational processes. They can be structured into two types:

- Approaches based on ordinal qualitative scales. Among them, the most notable ones are: (i) the approach based on ordinal scales and max-min operators [7], which was the first proposed approach using a linguistic scale $S=\left\{s_{1}, \ldots, s_{g}\right\}$ with a linear ordering as the only structure available in $S$; (ii) the approach based on convex combination [36], which directly computes on the indexes associated with the linguistic terms of the set $S$ recursively generating a real number on the granularity interval of $S$; (iii) the approach based on linguistic 2tuples [10], which uses a pair of values $(s, \alpha)$ in which $s$ is a linguistic term and $\alpha$ is a numeric value representing a symbolic translation to deal with ordinal linguistic information without loss of information; (iv) the approach based on virtual linguistic terms [37], [38], which extends the discrete term set $S=\left\{s_{-g / 2}, \ldots, s_{0}, \ldots, s_{g / 2}\right\}$ to a continuous one $S=\left\{s_{\alpha} \mid \alpha \in[-r, r]\right\}$ in which $r(r>g / 2)$ is an enough large positive integer (actually, this approach is similar to the approach based on linguistic 2-tuples from a mathematical computation point of view [38]); (v) the approach based on ordering proximity measures [39], which deals with non-uniform ordered qualitative scales; and (vi) the approaches based on multigranular linguistic term sets and linguistic hierarchies [40], [41], which uses linguistic terms belonging to different linguistic term sets with different granularity and semantics.

- Approaches based on linguistic scale functions, which try to solve decision-making processes by means of mathematical functions between linguistic terms and numerical values. Among them, the most notable ones are: (i) the approach based on proportional 2-tuples [42], which is based on the canonical characteristic values of the linguistic terms to deal with linguistic term sets that are not uniformly and symmetrically distributed; (ii) the approach based on numerical scales [43], which establishes a one to one mapping between the linguistic terms and a numerical scale; and (iii) the approaches based on specific mathematical functions that assign numerical values to linguistic terms to represent its corresponding semantic [44].

\section{F. Complex linguistic assessments}

The linguistic approaches shown in Section II-E assume the assessment provided by an expert consists in a single linguistic term chosen from a predefined linguistic term set. Even though they are appropriate to describe vague concepts associated with natural language, due to the expert's granules of knowledge and the complex decision-making processes [45], the employment of a single linguistic term might not be enough to express the expert's assessment. Actually, the need for selecting a linguistic term from a predefined set restricts the way in which the experts provide their assessment as the 
linguistic terms might not match the expert's opinion and, as a result, the expert must balance between several linguistic terms [46].

To avoid such a situation, the linguistic approaches should allow the experts to employ more than one linguistic term. This leads to the use of complex linguistic expressions, which allude to linguistic assessments that involve more than one linguistic term characterized by either artificial or natural languages via linguistic hedges, connectives and linguistic terms [46]. The use of complex linguistic expressions instead of single linguistic terms facilitates both the representation and elicitation of expert's assessment in a more elaborated way. However, dealing with complex linguistic expressions is considerably more difficult than managing single linguistic terms.

Several approaches dealing with complex linguistic expressions have been developed in the last decade (refer to [46] and [47] for a detailed description of them). The most notable ones are the approach based on hesitant fuzzy linguistic term sets [48] and the approach based on linguistic distribution [49].

1) Hesitant fuzzy linguistic term sets: They allow to model experts' hesitant assessment by means of comparative linguistic expressions (multiple successive linguistic terms).

Definition 7: [48] "Let $L=\left\{l_{0}, l_{1}, \ldots, l_{g}\right\}$ be an established linguistic term set. A hesitant fuzzy linguistic term set, $h_{L}$,

$$
h_{L}=\left\{l_{i}, l_{i+1}, \ldots, l_{j} \mid 0 \leq i \leq j \leq g\right\}
$$

is an ordered finite subset of consecutive linguistic terms of $L . "$

To better represent the complex linguistic assessments, the concept of double hierarchy hesitant fuzzy linguistic term set was introduced in [11]. Refer to [27] for recent developments of hesitant fuzzy linguistic term sets in decision-making processes).

2) Linguistic distribution: In comparison with hesitant fuzzy linguistic term sets, a linguistic distribution gives some symbolic proportion information over linguistic terms to characterize experts' distributed preferences as distributed assessments.

Definition 8: [49] "Let $L=\left\{l_{0}, l_{1}, \ldots, l_{g}\right\}$ be an established linguistic term set. A linguistic distribution over $L$ is defined by:

$$
D_{L}=\left\{\left(l_{i}, \rho\left(l_{i}\right)\right) \mid i=0,1, \ldots, g\right\}
$$

where $\rho\left(l_{i}\right) \geq 0$ is the symbolic proportion of $l_{i}$ and $\sum_{i=0}^{g} \rho\left(l_{i}\right)=1 . "$

Given a linguistic distribution $D_{L}, l_{i}$ indicates a linguistic term employed by an expert and $\rho\left(l_{i}\right)$ indicates the corresponding symbolic proportion information of $l_{i}$, as part of a probabilistic distribution associated with the linguistic terms [49]. Refer to [47] for a complete description of the linguistic distribution and its variants, i.e., proportional linguistic distribution, probabilistic linguistic term sets, incomplete linguistic distribution, interval symbolic proportions, possibility distribution for hesitant fuzzy linguistic term sets, proportional hesitant fuzzy linguistic term sets, and hesitant linguistic distribution.

\section{G. Other representation approaches far away from common} sense

In this section, we have recalled the fuzzy sets and its most used extensions to model experts' assessments in decisionmaking processes. However, in the literature, we can find other extensions of fuzzy sets that have been applied to decisionmaking [18], namely, Pythagorean fuzzy sets [50], picture fuzzy sets [51], q-rung orthopair fuzzy sets [52], probabilistic dual hesitant fuzzy sets [53], Fermatean fuzzy sets [54], to cite some of them. Even though they are mathematically correct, it has been seriously questioned whether constructions like those could actually be of any use in real decision-making processes asking to experts to use these fuzzy sets extensions to evaluate alternative preferences under a rational and realistic premise.

In decision-making, it is necessary to show that the representation domains used are useful from the vantage of the experts. However, constructions that are too complicated would not be useful because experts would be unable to express their assessments accordingly, as the interpretation of such constructions at times seems not to be well understood: they are far from common sense [55]. In such a way, they do not provide a significant enough contribution neither practically nor theoretically. Whenever a new representation domain is proposed, it is necessary to show that collecting such information would be realistic and actually useful in practical applications. Furthermore, once such a representation is established, it then needs to be shown that using it can effectively solve problems that currently arise.

\section{DECISION-MAKING FRAMEWORKS: PROBLEMS AND MODELS}

This section revisits three core decision-making scenarios, examining key characteristics, methodological trends, role of fuzzy and linguistic modelling in these scenarios, and some of their related challenges: multi-criteria decision-making (Section III-A), group decision-making (Section III-B) and multiperson multi-criteria decision-making (Section III-C).

\section{A. Multi-Criteria Decision-Making (MCDM)}

1) Framework description: In practical decision situations, it is infrequent that humans have a single criterion in mind when assessing an alternative. Decision processes guided by preferences elicited under a single criterion are therefore not sufficiently rational in many real contexts [3], [56]. MCDM methods help finding the optimal solution in such situations where evaluations need to be expressed under multiple (contradictory) indicators or criteria associated to the alternatives [57], [58].

A MCDM problem is formally characterized by:

- A finite set of decision alternatives, denoted $X=$ $\left\{x_{1}, x_{2}, \ldots, x_{n}\right\}, n \geq 2$.

- A finite set of criteria used to evaluate alternatives, $C=$ $\left\{c_{1}, c_{2}, \ldots, c_{q}\right\}, q \geq 2$.

- Each criterion $c_{j} \in C, j=1, \ldots, q$, has associated an importance weight $w_{j} \in[0,1]$. Criteria weights are usually normalized, i.e. $\sum_{j} w_{j}=1$ 
With these basic elements at hand, preference information is expressed in an evaluation matrix $M=\left(m_{i j}\right)_{n \times q}$, where each assessment $m_{i j}$ represents the evaluation given to alternative $x_{i} \in X, i=1, \ldots, n$, in accordance with criterion $c_{j}$. Thus, the two main inputs for a MCDM problem are (i) the evaluation matrix $M$, and (ii) the weighting vector associated to criteria, $W=\left\{w_{1}, w_{2}, \ldots, w_{q}\right\}$.

The existence of criteria weights naturally implies that in MCDM problems criteria may have diverse importance. Furthermore, there can coexist both qualitatively and quantitatively assessed criteria, both subjective and objective evaluations, as well as both benefit criteria (whose assessment values are to be maximized) and cost criteria (whose assessment values are to be minimized).

2) MCDM Approaches: Role of Fuzzy Sets and Extensions: Owing to the breadth of real-world application domains, the multidisciplinary character of MCDM research and the different motivations that may exist solving a MCDM problem, there exist various major approaches or families of methods to support these problems. Considering an operational viewpoint [57], each of these approaches assumes a different view to answer a common question: "how can we take into account all criteria comprehensively in order to compare potential actions (alternatives) between them?" [56].

The presence of uncertainty (vagueness, subjectivity, etc.) exhibited by humans in the preference elicitation process is another key aspect in practical MCDM scenarios. The necessity of handling such uncertain information in MCDM problems [69] has motivated the scientific community to develop a wealth of methods based on fuzzy sets, CW and their extensions, as well as other uncertainty theories. These developments have a double objective:

1) Representing uncertain preference information in MCDM. The representation scenarios examined in Section II are applicable in MCDM for modelling assessments. For example, for assessments expressed as fuzzy membership degrees we have $m_{i j}=\mu_{c_{j}}\left(x_{i}\right) \in[0,1]$, for assessments expressed as AIFS elements we have $m_{i j}=\left(x_{i}, \mu_{c_{j}}\left(x_{i}\right), \nu_{c_{j}}\left(x_{i}\right)\right)$, and so on.

2) Performing computational processes on uncertain information in MCDM, namely aggregation, comparison and distance/similarity measures between different forms of fuzzy sets, linguistic information, etc [8], [29], [33], [70].

Below we summarize the most prominent families of MCDM methods (see Table I) outlining their key features and highlighting the role of fuzzy set-based theories in these methods to apply computational processes on uncertain information.

(1) Value measurement methods are used in situations that require assigning a single assessment or utility value to each alternative in order to find the best alternative or establish a ranking of them. These methods have their roots in MultiAttribute Utility Theory (MAUT) [71]. One of its simplest approaches is the Simple Additive Weighting (SAW) method [59] in which, given $x_{i} \in X$, a weighted sum is used to aggregate $q$ assessments $m_{i j}$ into a representative value, $m_{i}$ indicating the overall intensity of preference for $x_{i}$, i.e. $m_{i}=\sum_{j} w_{j} \cdot m_{i j}$. An advantage of these methods is the ample repertoire of solutions tailored to handle different uncertainty representations [7], [72], [73] along with numerous approaches for the aggregation of information [72]. Aggregation [74] is a non-trivial process where several intricacies shall be considered beyond the compensation between high-low input assessments or the relative importance weights of criteria, e.g. inter-dependence between criteria [75], optimistic versus pessimistic aggregation attitudes [12], a mutual reinforcement effect of similar aggregation inputs [76], etc. As a result, a wide spectrum of aggregation operators have been established in the MCDM literature, implementing very diverse logics for aggregation processes, some of which accommodate fusing preference information under fuzzy and linguistic representations [77], [78]. A limitation in value measurement methods is the need for normalizing and unifying assessments before aggregating them. Another difficulty lies in the choice of aggregation function or selecting a suitable setting for criteria weights and other aggregation parameters.

(2) Reference level methods establish one or several reference points or "ideal solutions" and employ distance metrics with the goal of determining the relative position of every alternative with respect to these reference points, thereby identifying the most suitable alternative [61], [62]. Reference points represent either Positive Ideal Solutions (PIS) or Negative Ideal Solutions (NIS), such that the closer an alternative $x_{i}$ is to a PIS -and the farthest it is to the NIS, where applicable- the more suitable $x_{i}$ would be as a solution for the MCDM problem. Various widespread techniques under this category, e.g. TOPSIS, VIKOR, etc., differ from each other in the distance metric being used. Reference level methods stand out in their simplicity and adequacy to accommodate a large number of alternatives and criteria, compared to other families of methods. The choice of a distance measure between alternatives and reference points usually requires unifying cost and benefit criteria and normalizing assessments into a common scale. Some reference level methods may also exhibit limitations to distinguish between the performance of two or more alternatives. In order to cope with MCDM problems under fuzzy environments, extensions of reference level methods have been proposed whereby distance measures between fuzzy numbers, hesitant or intuitionistic fuzzy assessments, linguistic assessments, etc., are defined [79], [80].

(3) Outranking methods [63], [64], [65] abide to the principle of dominance between alternatives. These methods focus on comparing alternatives pairwise, predicated on a binary relation on $X$. Since outranking methods apply pairwise comparisons among alternatives at criterion level, they have the advantage of not requiring normalization or unification of assessments into a uniform representation, nor they are affected by compensation between criteria as aggregation-based approaches are. This implies that different fuzzy and linguistic representations can be used across distinct criteria, in which case extensions based on comparison laws between uncertain assessments can be used, e.g. [81]. On the contrary, they do not quantify how much more an alternative $x_{i}$ outperforms another alternative $x_{k}$, therefore they may incur loss of information.

(4) Pairwise comparison methods also compare alternatives against one another, but unlike outranking methods, they 
TABLE I

TYPES OF MCDM METHODS

\begin{tabular}{|l|l|l|}
\hline Type & Key features and computational processes in fuzzy/linguistic extensions & Representative methods \\
\hline Value Measurement & $\begin{array}{l}\text { Easier to compare with other MCDM methods. They require normalizing } \\
\text { into a uniform assessment representation and scale. Aggregation as key } \\
\text { computational process in fuzzy/linguistic extensions. }\end{array}$ & $\begin{array}{l}\text { Simple Additive Weighting (SAW) [59]; } \\
\text { Weighted Aggregated Sum Product Assessment } \\
\text { (WASPAS) [60] }\end{array}$ \\
\hline Reference Level & $\begin{array}{l}\text { Finding alternative(s) that is/are closest to an "ideal solution" and/or } \\
\text { farthest from a "negative ideal solution". Simpler to apply than other } \\
\text { families of methods. They require normalizing assessments. Distance } \\
\text { measurement as key computational process in fuzzy/linguistic extensions. }\end{array}$ & $\begin{array}{l}\text { Technique for Order Preference by Similarity } \\
\text { to Ideal Solution (TOPSIS) [61]; Multi- } \\
\text { ple Criteria Optimization Compromise Solution } \\
\text { (VIKOR) [62] }\end{array}$ \\
\hline Outranking & $\begin{array}{l}\text { They do not require normalizing nor unifying assessments. Can not quan- } \\
\text { tify how much one alternative is more preferred than another. Comparison } \\
\text { as key computational process in fuzzy/linguistic extensions. }\end{array}$ & $\begin{array}{l}\text { Preference Ranking Organization METHod of } \\
\text { Enrichment Evaluation (PROMETHEE) [63]; } \\
\text { Organisatioon, Rangement Et Synthèse de } \\
\text { données relaTionnElles (ORESTE) [64]; EL- } \\
\text { limination Et Choix Traduisant la REalite } \\
\text { (ELECTRE) [65] }\end{array}$ \\
\hline Pairwise comparison & $\begin{array}{l}\text { They can reflect how much one alternative is more preferred than another. } \\
\text { Analytic Hierarchy Process \& Analytic Network } \\
\text { Process (AHP, ANP) [66] }\end{array}$ \\
\hline Mixed/other & $\begin{array}{l}\text { Mixed methods combine the strengths of multiple approaches. Goal } \\
\text { programming as multiple-goal extension of linear programming models. }\end{array}$ & $\begin{array}{l}\text { Multi-Objective Optimization on the basis of Ra- } \\
\text { tio Analysis plus full multiplicative form (Multi- } \\
\text { MOORA) [67]; Goal Programming [68] }\end{array}$ \\
\hline
\end{tabular}

are used when it is necessary to reflect how much more preferred/suitable is $x_{i}$ against $x_{k}$. AHP and ANP, along with extensions to compare fuzzy information, are some of the most popular classes of methods under this approach [66]. They have consequently more expressive power than outranking methods and thus they have been deeply investigated from the viewpoint of modelling fuzzy and linguistic information [82] to express strengths of preference, although they lead to inherently more complex process for the same reason.

(5) Mixed/other approaches: Lastly, there exist mixed approaches like MULTI-MOORA [67] that combine aspects from various approaches, e.g. value measurement and reference level mechanisms. Other approaches like Goal Programming to optimize multiple objective functions, have been also considered in MCDM research [68], both standalone or combined with other methods.

3) Challenges of fuzzy sets and their extensions in MCDM: Despite their proliferation across the area of MCDM, the overall application of fuzzy sets, $\mathrm{CW}$ and their variants in MCDM should be thoroughly revisited and put in perspective, in line with current developments in Artificial Intelligence (AI), Big Data and related paradigms that can potentially help dealing with increasingly larger and more complex decision problems. Some challenges to be considered are listed below:

- Recent substantial advances in Natural Language Processing (NLP) would enable the extraction of accurate evaluation information from human text [17] or speech. These advances should be embraced for bridging the gap between NLP and fuzzy decision-making scenarios in a way that brings future developments back towards human common sense. Concretely, in MCDM it is necessary to start dealing with richer and less restricted human-like linguistic expressions that not only capture assessments on alternatives /criteria but also describe interrelationships between these.

- Data-driven methodologies could help extracting (sometimes objective) insight about opinions, depending on the nature of data [83]. Yet, the emergence of these paradigms should not necessarily mean replacing the (usually subjective) elicitation of assessments from a hu- man expert, but rather investigating the synergy between both sources, exploring how their interaction could help handling uncertainty effectively or even dispelling it. Besides, in some cases, the criteria are not independent but interacting, which indicates the necessary to extend data-driven methodologies in MCDM by considering interactions among criteria. Data-driven decision support (see Section IV-F) is important for many businesses and has been discussed in many MCDM problems, such as preference discovery, but it still needs the verification and application for data-driven methodologies, which are required to be more exhaustive and extended.

\section{B. Group decision-making (GDM): Preference Relations}

1) Framework description: Making collective decisions implies the joint participation of several experts in a decision problem, each of whom have their own ideas, attitudes, motivations and knowledge. Group decisions often lead to better and less biased solutions than those made by a single person [84], [85] but they intuitively introduce the added complexity of how to effectively combine individual preference information into collective information for making adequate (and acceptable) decisions.

A GDM problem is formally characterized by a finite set $X=\left\{x_{1}, \ldots, x_{n}\right\}$ of $n \geq 2$ alternatives, and a group $E=\left\{e_{1}, \ldots, e_{z}\right\}$ of $z \geq 2$ participants or experts, who assess alternatives in $X$ and attempt to find a common collective solution for the problem. Similar to MCDM, some GDM approaches assign different importance weights $\lambda_{k}$ to experts $e_{k}, k=1, \ldots, z$. Frequently, in GDM problems each expert $e_{k} \in E$, expresses her/his opinions in $X$ via pairwise comparison assessments between the available alternatives, concretely by providing a preference relation. Orlovsky studied in [86] the properties of fuzzy preference relations. A fuzzy preference relation associated with $e_{k} \in E$ on a finite set $X$ of $n \geq 2$ alternatives, is given by a fuzzy binary relation and represented as a $n \times n$ matrix $P_{k}=\left(p_{k}^{i j}\right)_{n \times n}$ of assessments $p_{k}^{i j}=\mu_{P_{k}}\left(x_{i}, x_{j}\right) \in[0,1]$. In accordance with the notions introduced in Section II-B, each assessment indicates the strength of preference by $e_{k}$ towards alternative $x_{i}$ when 


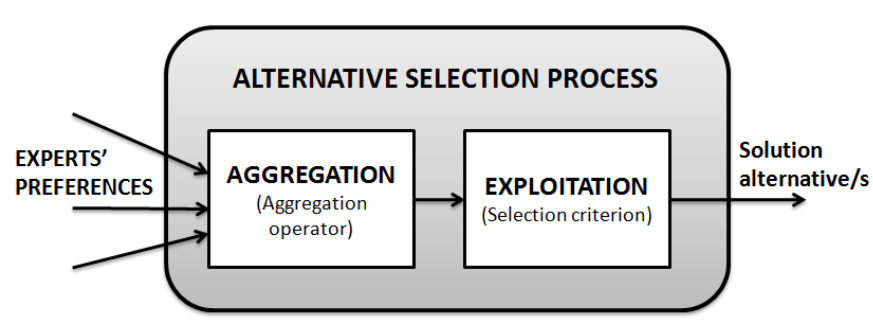

Fig. 1. Selection process for solving GDM problems. Source [85]

compared against $x_{j}(i \neq j)$. Concretely, if $p_{k}^{i j}>0.5$ then $x_{i}$ is preferred over $x_{j}$ by $e_{k}$. If $p_{k}^{i j}<0.5$ then $x_{j}$ is preferred over $x_{i}$ by $e_{k}$, and if $p_{k}^{i j}=0.5$ then both $x_{i}$ and $x_{j}$ are deemed as indifferent or equally preferred.

The solution for a GDM problem typically consists of two stages, as illustrated in Figure 1 [84], [87]: (1) an aggregation phase in which the individual experts' preferences are combined at assessment level by using an aggregation operator [74]; (2) an exploitation phase where the best alternative(s) as the solution to the problem are identified, or a ranking of them is established, e.g. using a dominance criterion [86].

2) Consensus and Uncertainty Management in GDM: Motivated by the possibility that some experts might exhibit disagreements with each other's opinions or they may sometimes not accept the decision made, Consensus Reaching Processes (CRP) were introduced as a key requirement in GDM problems to achieve a high level of collective agreement before the alternative(s) selection process [9], [88], [89], [90], [91]. Achieving consensus requires that some experts modify their initial opinions, bringing them closer to each other, towards a collective opinion deemed as satisfactory by the group. Figure 2 shows a general iterative scheme followed by most existing approaches for CRPs in the literature. Its main stages are: (1) Consensus measurement, i.e. determining the current closeness level between opinions by using a consensus measure [49], [92]; (2) Consensus control to determine whether the desired level of consensus has been achieved; (3) Consensus progress, applying a feedback mechanism to bring farthest opinions closer to the rest of the group and increase the level of agreement [88], [89], [90], [93].

In general, in a CRP experts manually modify their preferences guided by a feedback mechanism [94]. However, as stated in [91] this manual process may incur laborious negotiations and a prohibitive cost, under a limited resources setting. Automatic feedback mechanisms based on minimum adjustment/cost have consequently arisen to support efficient consensual decisions [89], [95], [96], [97].

In terms of uncertainty management in GDM, in parallel with developments made on preference representations in fuzzy and linguistic contexts, researchers have also devoted efforts in defining GDM models based on diverse classes of preference relations [24], [49], [98], [99] in which assessments can be modelled by AIFS, hesitant fuzzy sets, simple and complex linguistic expressions, etc. Likewise, some GDM models incorporate fusion mechanisms to integrate assessments expressed in different formats (heterogeneous information), accommodating groups of experts with different

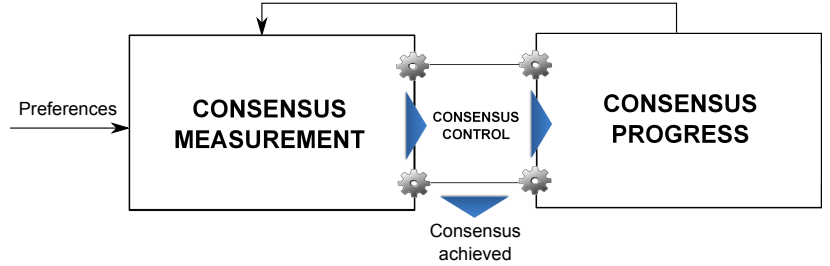

Fig. 2. General scheme for CRPs. Source [90]

expertise or uncertainty levels [100], [101]. CRP models for managing different forms of uncertainty [16] have been also proposed, with specifically designed consensus measures, feedback mechanisms and minimum cost models for this end, as well as fuzzy interpretations for the concept of majority in CRPs [102].

3) Recent trends and challenges in GDM: As a general rule, most of the recently investigated challenges in MCDM problems -e.g. for gathering, representing and applying computational processes on preference information- as well as presently open questions, also apply to GDM situations. In addition, societal and technological advances in the last decade have led to major challenges inherent to GDM problems in general and consensus-driven ones in particular, including:

- Modeling social relationships in GDM, by integrating and exploiting relationship information e.g. trust between experts, in GDM problems and using them to guide CRPs [15], [103], [104].

- Large-scale GDM problems [85], [105], in which decision groups become larger, more diverse, and additional difficulties arise such as the necessity of clustering a large group into subgroups or identifying strategic manipulative behaviors that may bias the CRP [16], [104], [106], [107], among others.

- Opinion dynamics in GDM, a close concept to social network GDM according to which individual opinions evolve influenced by other experts' opinions [108].

- Conflict resolution [109], focused on analyzing relationships and/or opinions of experts for detecting and eliminating conflicts between them.

- Personalized Individual Semantics [110], which occurs in GDM problems where preferences are assessed linguistically or under the $\mathrm{CW}$ paradigm, and each group member has a different semantic interpretation for the linguistic terms or expressions being used.

Some of these challenges have received a lot of scientific attention in recent years, thus leading to the development of new trends and complex decision-making paradigms. Accordingly, they are discussed in more detail in Section IV.

\section{Multi-person Multi-criteria decision-making (MpMcDM)}

1) Framework description: There are decision processes that consider the co-existence of multiple experts and multiple evaluation criteria. Formally, these processes are defined by a set of alternatives $X=\left\{x_{1}, \ldots, x_{n}\right\}$ that are evaluated by a group of experts $E=\left\{e_{1}, \ldots, e_{z}\right\}$, according to a set of criteria $C=\left\{c_{1}, \ldots, c_{q}\right\}$, with $n, z, q \geq 2$. Models for 


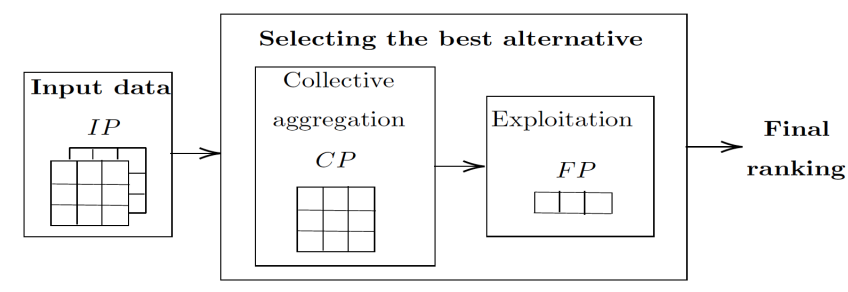

Fig. 3. Traditional MpMcDM workflow. Source [17]

supporting these processes are known as Multi-person Multicriteria decision-making (MpMcDM) models [111], [112].

The majority of MpMcDM models under a fuzzy context in the literature consider two main approaches: (i) expert aggregation followed by criteria aggregation [113], [114], or (ii) criteria aggregation followed by expert aggregation [115]. The first of these two approaches is the most extended in $\mathrm{MpMcDM}$, and its workflow (see Figure 3) is divided into two phases [17]:

- Input data phase: Experts $e_{k} \in E$ provide their evaluations about the alternatives, in the form of $m$ individual decision matrices $M_{k}=\left(m_{i j}^{k}\right)_{n \times q}$.

- Selection phase: The individual assessments of experts are aggregated into a collective evaluation matrix $M=$ $\left(m_{i j}\right)_{n \times q}$, with $m_{i j}=\phi\left(m_{i j}^{1}, \ldots, m_{i j}^{q}\right)$ and $\phi$ an aggregation operator. Assessments in the collective matrix are then aggregated across criteria, yielding a preference vector or a ranking of alternatives in $X$.

Individual evaluations can be expressed through numerical ratings, linguistic variables, or extensions of fuzzy and linguistic representation models (see Section II). Furthermore, consensus processes are often introduced in some $\mathrm{MpMcDM}$ studies, in which case they usually take place before the aggregation workflow described above [104].

2) Challenges in MpMcDM: Since MpMcDM problems combine the characteristics and complexities of both MCDM and GDM problems, the previously described challenges in both scenarios apply to MpMcDM too. Nonetheless, additional examples of challenges and difficulties that predominantly occur in MpMcDM are:

- Subjective criteria priorities: Subjectivity has been widely investigated to help eliciting preferences in fuzzy settings, but very few studies such as [116], [117] have tackled problems where different experts might exhibit different perspectives about the relative importance of criteria, in which case a single criteria weighting vector for the whole group becomes unsuitable. Due to its complexity, this aspect deserves further research.

- Psychological aspects such as prospect theory could help better understand experts behavior and rationality particularly when preferences are expressed linguistically [118], however these aspects have barely been investigated in MpMcDM, MCDM and GDM models.

\section{Challenges IN DECISION-MAKING GUIDED BY WISDOM OF THE CROWDS}

This section discusses emerging complex decision-making paradigms in recent years, providing a critical reflection about the advances made, prospects, challenges and opportunities brought along by current societal and technological developments. Throughout the discussions, we also elaborate on the urging need for rethinking various aspects in the state of affairs of fuzzy and linguistic decision-making as a whole. The challenges are organized into several emerging scenarios and paradigms, as shown in Figure 4.

\section{A. Consensus: what is next?}

1) Description: As previously seen, consensus reaching is arguably the most investigated research trend within GDM, and also a popular topic in MpMcDM to some extent, with hundreds of published studies today from management, computer science and other disciplines. An overview and taxonomy of consensus models under fuzzy (and linguistic) contexts was presented in [90], highlighting the different types of consensus measures and feedback mechanisms to guide CRPs. Subsequently, the review in [88] deepens its analysis into CRPs guided by a moderator and based on the concept of fuzzy majority to model the semantics of consensus in a human-consistent manner. In [119] CRP methods in social network GDM scenarios (see Section IV-C) are investigated, whereas the book in [85] describes the stateof-the-art consensus approaches for supporting large-scale decisions (see Section IV-D). More recently, the overview in [91] focuses on analyzing feedback mechanisms based on minimizing preference adjustment cost in CRPs, examining the mechanisms developed to this end in both classical GDM problems and complex ones (large-scale, trust-based, opinion dynamics-driven etc.).

The above surveys, all of which have been undertaken along the last seven years, show the wealth of studies in the last decades devoted to improve CRPs in a variety of decision contexts: different preference structures and representation formats, heterogeneous groups where experts have different importance levels, consistency-preserving, adaptive and agentbased CRPs, emergency decision scenarios, etc. These works also highlight a number of challenges and future directions in the field, some of which still remain unaddressed today.

2) Challenges: The progress made in developing CRP solutions has also given prominence to challenges and open questions, whose answer could help taking important steps forward in this research area in terms of advancing practical and real world solutions that enable fair and socially accepted community decisions by crowds. Below we discuss two of these challenges deserving scientific attention hereafter:

(I) A (much needed!') paradigm shift towards practical and applicable CRPs: Compared to the vast theoretical developments made in defining consensus models, there is a worrying and very unbalancing absence of efforts focused on the evaluation, validation and comparison of CRP solutions as well as their deployment in real applications. Rather alarmingly, this means that despite the hundreds of consensus models in the literature, 


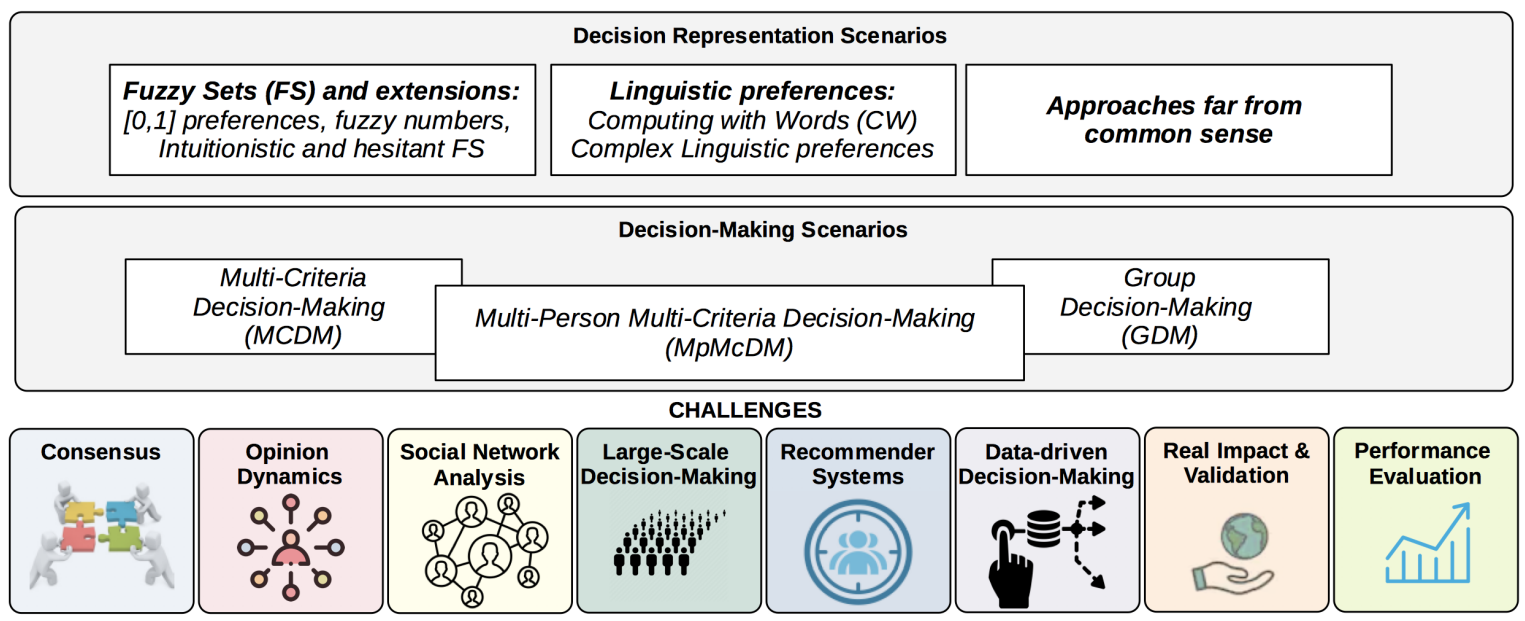

Fig. 4. General outlook on preference representation scenarios, decision-making scenarios and challenges for making better decisions guided by crowds

little real progress has been made from a practical point of view. We previously denounced the increasing surge in representation and computational models based on non-trivial fuzzy set/CW extensions, aggregation models, etc., that (i) are going beyond the limits of human common sense, and (ii) have little or no contribution in usability for decision makers in real problems. Still, unfortunately these issues are no exception in most CRP proposed methodologies. Future research should no longer prioritize more and more extensions of models that resemble each other, but rather enable the experimental validation and practical use of these models: "in real world, by real people". A first mandatory step for this is to investigate comprehensive cost and performance metrics alongside other desirable CRP properties beyond covergence/cost, to evaluate and compare different consensus models in a realistic and standardized fashion. In the case of CRPs guided by minimum cost adjustments, it also worth examining the fact that opinion modification costs may have individual interpretations, as suggested in [91]. Ultimately, with the rise of social platforms, AI and reproducible data-driven research paradigms, we believe now it is the right time for the research community to initiate a drastic paradigm shift into a more practical viewpoint. Further and more general guidelines in this direction are discussed in Sections IV-G and IV-H.

(II) Personalized feedback strategies and gamification: In CRPs where experts receive feedback and manually adjust their initial opinions, they can sometimes decline such feedback and hamper the process. Many studies [16], [104], [107] resort to penalizing the influence of these experts, but none so far have considered a completely "opposite" perspective consisting in finding ways to motivate experts' willingness to bring their individual opinion closer to the rest of the group. A possible starting point in this direction could be the development of personalized feedback mechanisms where, for instance, if there exist several assessments requiring adjustment by a given expert, the element(s) she/he would be less reluctant to adjust may be identified. Meanwhile, a recent study [120] also hints at the potential benefits of gamification as a driving force to increase experts' engagement in the CRP through the feedback mechanism, and highlights the necessity of bridging the gap between these ideas and game theory in future research.

Other challenges inherent to large-scale, social network decision-making, real-world validation and application of models, etc. -some of them applicable to consensual decisions- are described in the following subsections.

\section{B. Social Networks for Crowd decision-making: Opinion Dy- namics}

1) Description: With the development and evolution of communications networks, social relations among people become more convenient but complex: this phenomenon promotes the emergence of a new type of social networks for crowd decision-making. Social networks provide a platform for users to share information and communicate with each other, and therefore shows the social relationships among them. During interactions, the opinions of experts would be influenced by others in a social network, which makes such opinions dynamic. Opinion dynamics describe the process of updating opinions of interactive experts in an iterative and interactive process, leading to consensus, fragmentation or polarization of opinions. [121]. The evolution of opinion dynamics usually consists of three key elements:

- Opinion expressions formats: For different experts, they would have different preferences and therefore apply different opinion expressions formats, such as discrete opinions, continuous opinions, and uncertain opinions.

- Evolution rules: In social networks, the experts would follow the evolution rules rather than changing their opinions randomly, where the evolution rules provide the directions for the experts to change and update their opinions repeatedly.

- Opinion dynamics environments: Under the decision making environments, the opinions of experts form a stabilized evolution result: consensus, fragmentation or polarization.

A review of opinion dynamics is presented in [108].

2) Challenges: Although opinion dynamics in social networks for decision-making have been investigated widely, there still exist some challenges: 
- Bridge the gap between opinion dynamics and GDM: The research of GDM develops methods to make a consensus decision by aggregating experts' opinions. Both opinion dynamics and GDM study the opinions formation under a group environment. The key points are how to bridge a gap between opinion dynamics and GDM, to study the connections between them, and to apply classical GDM methods in opinion dynamics contexts.

- Characteristics of linguistic Opinions: It is an inevitable trend to study crowds decision-making based on opinion dynamics in linguistic decision-making contexts. Particularly, experts would apply different linguistic expressions formats, and there exists the fact that words mean different things to different people, which implies the necessity to take into account the characteristics of linguistic opinions and the personalities among experts.

- Strategic manipulation with opinion dynamics: As mentioned before, the evolution of opinion dynamics in social networks generally forms three stabilized status: consensus, fragmentation or polarization, all of which can be manipulated strategically to achieve a preset result. The key point is how to discover and develop the guiding patterns for managing the evolution of opinion dynamics.

- Opinion and action dynamics: In social networks for decision-making, the evolution of opinions would influence the actions or choices of experts, which further changes the structures of the social network. And in real problems, there would be always the situation that the opinions and actions dynamically change in social network, which makes it difficult to make accurate decisions.

\section{Social Network Analysis-based decision-making}

1) Description: Social network analysis (SNA) provides a powerful tool to study the relationships among users (individuals, groups or nations) in social networks for decisionmaking, which can examine the properties including centrality, prestige, structural balance and trust relationship [97]. In SNA, consensus processes based on trust relationships have been widely investigated in existing studies, which mainly from the following three aspects:

- Preference aggregation driven by trust relationships: It studies the aggregation of individual preferences based on aggregation operators to obtain a collective preference, where the importance degrees of experts, if unknown beforehand, are computed from trust relationships based on the general assumption that those experts more trusted by other experts should have higher weights.

- Incomplete preference estimation based on trust relationships: It estimates missing/unknown preferences of an expert based on the preferences provided by other experts he/she trusts.

- Feedback recommendation guided by trust relationships: In SNA, it is common that an expert would be more willing to change his/her preferences if the suggestions are from the experts he/she trusts, which makes feedback processes more persuasive and realistic.
A review of social networks analysis based decision-making is presented in [15].

2) Challenges: SNA based decision-making has attracted a lot of attention, but there are still some challenges:

- Identify real trust relationships: Trust relationships play an important role in SNA based decision-making, but there is always the case that real trust relationships or degrees between experts are unknown or are not easy to identify when starting the decision process or are dynamically changing. The challenge is how to develop methods to identify real trust relationships among experts instead of using pre-established ones.

- Dynamic SNA: The consensus in SNA driven by trust relationships was widely investigated in the existing studies, including the preferences aggregation, the estimation of incomplete preferences and the modification of preferences in feedback recommendation, while these studies assume that the trust relationships are static. However, in real-life decision-making, the trust relationships are also changing dynamically as dynamic opinions do, which indicates the necessity of the consideration of the dynamic trust relations and also the influences to the final decision result.

- Dishonest behaviors in SNA: A core issue during the decision-making process is that the experts may be dishonest, and they may express their preferences strategically by covering up their real thoughts, in order to achieve their goals. So the difficulty lies in detecting the dishonest behaviors in opinion expressions and social relationship formation in SNA.

\section{Large-Scale decision-making: Intelligence of the Crowd}

1) Description: A surge in societal consciousness-raising on democratic matters along with the rise of social media, cloud and e-government platforms, is stimulating a shift from conventional small-group GDM towards participatory events at large scale [85], [105]. This socio-technological shift has caused Large Scale decision-making (LSDM) scenarios to become more and more popular. A growing number of LSDM studies and methodologies have been published along recent years, the earliest of which extend conventional GDM or MpMCDM approaches by introducing innovative features.

LSDM problems are inherently different and more complex events than GDM in various ways, with some key nuances that are intrinsic to LSDM situations:

- Number and nature of participants: Whilst classical GDM and MpMcDM problems typically consider a small number of experts, LSDM situations contemplate scenarios where tens to thousands of individuals can participate. Managing such large groups often requires clustering participants into smaller subgroups so that the process is more manageable [16], [106], [110]. Furthermore, although small-group decisions usually involve domain experts, stakeholders or directives whose background is closely linked to the scope of the decision problem itself, in an LSDM problem participants might not only be specialized stakeholders but also entire communities 
potentially affected by the decision, whose members' background and levels of expertise may greatly vary.

- Impact: Large-scale decisions are usually important for society or certain communities of people, e.g. the inhabitants in a city, hence they should not exclusively involve the participation of a few representative experts/stakeholders, but rather accommodate the presence of as many affected individuals by such decisions as possible in the decision-making process.

- Acceptance of decisions: To guarantee the understanding, acceptance and support of the decision by as many participants as possible, as well as to reduce conflicts among them, it is often imperative to apply CRPs [90] as well as detecting and eliminating conflict relationships [109] within a limited number of iterations. Both CRPs and conflict elimination processes turn LSDM into highly dynamic and iterative processes.

Based on the aforesaid characteristics that describe LSDM scenarios, Ding et al. provided in [105] an unified definition for LSDM that also assumes that alternatives are described under several criteria, due to their complex nature.

Definition 9: [105] An LSDM event is an event where a finite set of alternatives are judged or assessed under several criteria, at least 20 participants are invited to take part, and the process is dynamic and aimed not only at selecting the final decision, but also at increasing the support shown by participants and/or decreasing objections to such a decision.

2) Challenges: Recent studies show that AI-based tools have been used in a large number of LSDM methodologies to support various of the above complexities and other ones [85], for instance: clustering algorithms to identify subgroups, detection approaches to identify and manage noncooperative/strategic behaviors, agent-based theory to emulate human behavior, social network analysis to explore relationship and trust patterns between participants, and data visualization tools to monitor the process status, among others.

Nevertheless, there are still ongoing challenges and unaddressed research questions that deserve further attention in the LSDM literature, particularly with regards to the long-term ambition of turning LSDM processes into highly participatory and crowd-driven phenomena, i.e. enabling events guided by the "intelligence of the crowd". Some of these challenges are summarized below, highlighting the crucial role to be played by AI, emerging technologies and data-driven approaches:

- Data and Knowledge Integration: It is no longer realistic to model large-scale collective decisions as the byproduct of subjective judgement information provided by participants solely. These decisions are very rarely made by communities of people who are socially isolated or independent from each other. Instead, different forms of social relationships -trust, distrust, influence, reputation, or even antagonism- coexist, hence SNA-based decision-making (Section IV-C) acquires special relevance in large-scale settings [97], [15]. Besides, profile or historical information associated to participants might have a great potential not only to better understand their background, expertise or priorities over the evaluation criteria, but also to learn suitable parameter settings for the LSDM model being utilized, or even to deal with -or eliminate- uncertainty and incompleteness. The main difficulty lies in making such participant-related data sources readily available for LSDM models or platforms, to analyse and extract valuable knowledge from them using AI.

- Analysis Dashboards: As LSDM problems become more scalable and capable of accommodating larger crowds, and additional data about participants and alternatives become available, more research is needed for effective visualization tools capable of accommodating Big Data in this context. For instance, analysis dashboards for monitoring and getting insight from diverse aspects of the LSDM problem, participants, performance and outcomes.

- Blockchain for distributed, safe and transparent LSDM: Online participatory decisions at large scale raise the demand for decentralized infrastructures that enforce security, integrity, transparency and cost-effectiveness, at the same time as the necessity of a central authority (moderator) is removed. Blockchain technologies would therefore play a key role in the real-world deployment of decentralized LSDM systems driven by the crowd, also enabling traceability in decision processes [122].

- LSDM in Smart Territories and 'Internet of Things' (IoT): Active information sharing between connected IoT devices and sensors in smart territories -a broader conceptualization of smart cities- can ultimately influence LSDM processes when coupled with contextual machine learning or other AI approaches to obtain knowledge about the context surrounding participants. Investigating information fusion techniques for modelling some aspects of the "crowd intelligence" behind LSDM problems, is still an unexplored challenge [123].

\section{E. Recommender System-based decision-making}

1) Description: Recommender Systems (RS) are presently a popular data-driven decision support tool to filter information for users in a personalized fashion, matching their preferences, interests or needs [124]. RS are used in diverse Internet platforms and domains in order to overcome the Information Overload problem, e.g. e-commerce portals, media, entertainment and tourism platforms, etc. In essence, an RS analyzes user-system interaction data -most commonly ratings given by users to items in the system- and learns about the user's preferences in order to predict items the user might be potentially interested in. Most recent RS models undertake this task predicated on AI and machine learning algorithms. A great deal of research within the RS scientific community has been devoted to two families of approaches whose basic principles are inspired by GDM and MCDM models:

- Group RS: Recommendation approaches for groups of users are popular in areas such as tourism, leisure and entertainment. Group RS rely on fusion methodologies to combine individual preferences (ratings) into a group preference representation, or merging individual lists of recommended items into a representative list of recommendations for a group [125]. 
- Multi-criteria RS: Similar to MCDM scenarios where options are evaluated under several criteria, in a multicriteria RS users rate items (e.g. hotels) using several criteria (e.g. food, cleanliness, service), hence these systems can aggregate and exploit such ratings [126].

2) Challenges: Although decision-making scenarios have inspired a number of models for group and multi-criteria RS, further research is still needed in two directions.

Firstly, in line with the rising demand for large-scale decision support tools based on LSDM approaches, there is also an growing need for RS approaches capable of accommodating larger groups of users. Future research at the intersection between RS and LSDM would have an impact on users' experience in large-group or mass events, e.g. group tours, advertising and music playlists at gyms, public venues, sports matches, etc., making the aforesaid experience more personalized. Clearly, a vital requirement to make this possible is the availability of information sources (ratings, social media data, and so on) associated to attendees in large-scale events.

Secondly, data-driven decision-making (described in detail in the next subsection) opens new research avenues whereby opinions can be objectively modelled upon data associated to experts. For example, the idea of leveraging opinion mining from individuals' data in the Internet using sentiment analysis has been recently investigated in [17]. Accordingly, extracting and integrating expert/crowd opinions from Big Data that reside in RS platforms -e.g. by mining text reviews and ratings for items- is worth further exploring to estimate evaluations on decision alternatives and criteria.

\section{F. Data-driven decision-making for smarter Decision Aid}

1) Description: Nowadays, decision support is increasingly studied in large-scale domains with vast amounts of diverse data instead of experience. These data, reflecting the available information and states of problems, reduces uncertainty and provides basic and sufficient support for decision-making. Therefore, data-driven decision support has become an important tool in decision-making to aid experts in making smarter decisions by analyzing data. Machine learning provides a powerful tool for supporting data-driven decisions, based on which data-driven decision-making [17], [127] has been applied across businesses.

2) Challenges: Data-driven decision-making provides a new decision paradigm for smarter decisions, but there still exist some challenges:

- Extract useful information from diverse data: In datadriven decision-making, the datasets including opinions or reviews, numerical information, sounds, pictures, etc., are generally collected for comprehensive and multidimensional analyses, as well as to extract assessments from such data e.g. by using NLP [17]. How to extract valuable information from different kinds of data to achieve useful information is an issue that needs to be further explored and identified. Besides, in linguistic decision-making contexts, personalized individual semantics and emotional factors can affect the final decisionmaking results. The difficulty lies in discovering the individual difference in semantic understanding of words and analyzing the true thoughts of experts.

- Data-driven LSDM: LSDM problems widely exist in our real world. Under a large-scale context, the decisionmaking methods, the related data and the social network structures would be different from that in traditional decision-making. The challenge is how to analyze and utilize the characteristics of LSDM and to make decisions under the data-driven LSDM context.

- Preference learning: The learning of predictive models, such as preference learning and semantic learning, has been a prevalent topic in data-driven MCDM. Some research results have been achieved in analyzing the characteristics and learning functions of individual preferences and collective preferences. However, real-life decisionmaking involves diverse data, which makes it much more complex to dig out the preference learning models. In addition, the influence of personalized individual semantics among experts in data-driven decision process should not be ignored.

- Data and AI integration: Nowadays, the combination of Big Data, IoT and AI brings opportunities and challenges to data-driven decision-making. The key point is how to integrate AI technology into data analysis and social network studies, to further apply data-driven theory and methodologies into practical decision-making problems for decision aid.

\section{G. On the need of Real and Practical Case Studies to validate the Real Impact of Models}

1) Description: decision-making methods in the literature are, in most cases, illustrated through step-by-step "application" examples or cases studies, although reality shows that it is relatively infrequent to find evidence of real-world validation, "real" applications or experimental comparisons against other baseline methods in these studies. Comparisons and highlights of a newly proposed model against existing ones, are typically done at a theoretical level only, in the form of a plain and mere comparison between the key features of two or models, and without experimental studies aimed at benchmarking several models. Most importantly, these pseudocomparisons fail to provide or consider objective evaluation metrics to analyze and discuss the practical performance of each solution being compared. Asking questions like "what property(es) are we pursuing to achieve with the use of this decision-making model?" are vital to identify appropriate metrics and evaluation standards, as detailed in IV-H next.

2) Challenges: In new studies, specially those focused on supporting complex decision-making scenarios such as those described in previous subsections, it is not enough to justify the novel contributions made in new studies per se: their impact and practical value for individuals, groups or crowds facing decision problems should be justified. We postulate that the shortage of experimental contributions in this sense is mainly due to (i) the absence of real, shareable datasets describing human opinions or instances of real decisionmaking problems, as well as (ii) the lack of standard evaluation 
methodologies to objectively assess and compare models -as previously hinted for the case of CRP models (Section IV-A).

Future research should move towards data-driven and reproducible decision-making solutions predicated on software model implementations and data sharing (under ethical considerations and regulations where applicable). This could facilitate and incentivise a new and much needed paradigm shift across the scientific community, towards more practical and applicable solutions closer to real-world situations.

\section{H. Evaluating the Performance of decision-making models}

1) Description: validating the impact and value of decisionmaking models in real scenarios also requires a prior evaluation of their performance in terms of desirable quality properties. A small number of studies paid some attention to this necessity, for instance the AFRYCA framework that implements several consensus models for their comparison [90] and the GDM-R framework built in $R$ that accommodates missing assessments in CRPs [128].

2) Challenges: Evaluating the performance of MCDM, GDM and MpMcDM approaches is still a very open, vaguely defined and complex challenge, even though this is a wellstructured and matured aspect of study in areas like machine learning, bibliometry, recommender systems and optimization methods, to name a few. It is not surprising in these areas to even find scientific competitions focused on setting points of reference for evaluation methodologies [129], yet in decisionmaking there is a serious gap in this aspect, as a consequence of which myriad studies are being published without truly undergoing a properly justified practical validation of their methodologies. It is imperative to start adopting solid analysis criteria to assess model performance based on the following three requirements:

1) Ground-truth, i.e. collected evidence in the form of data that assure the veracity of solutions (decisions) returned by models. To the best of our knowledge, there are no works focused on investigating this issue. An added complexity to the strain of making this ground truth available (e.g. logging previous real-world problem preferences and the outcomes of the decision made), is the necessity of adhering to common sense uncertainty representations in such data-something that most recent publications in the field have left behind.

2) Software libraries that implement state-of-the-art models and facilitate experimental comparisons, similar to what has been done in optimization [129]. There are almost no studies addressing this gap, albeit two initial approximations were presented in [90], [128].

3) Standardized quality measures are also virtually nonexistent. In a decision-making process, the notion of quality may vary from one decision scenario to another (see Section III), but some common guidelines on quality properties to consider are still needed for each scenario and type of decision problem: e.g. quality of the ranking/ordering of alternatives, separability or discriminatory power, consensus level achieved and cost to achieve it, etc.
On a last remark, the lack of well-defined guidelines for the performance evaluation of decision-making models is causing a serious and rather undesirable "relaxation" in terms of published studies whose actual contribution might be insufficient.

\section{Other challenges and open problems}

In preceding subsections we covered decision scenarios and paradigms that have gained a key position in fuzzy decisionmaking research at present. Without intending to be exhaustive, we finalize outlining other open problems in the area, of course, connected with the previous areas.

1) Large-scale social network with dynamic opinions: In social networks, the opinions of individuals would be easily influenced by the opinions from others. For example, in political elections, the evolution and prediction of opinions are key issues, and the evolution process may be intervened or manipulated. The theory and methodology of opinion dynamics in large-scale social networks has been widely studied, but there are still lacks in the practical application of decisionmaking problems.

2) Detecting and influencing opinions: In real life decisionmaking, there are many methods for detecting and influencing the individuals' opinions. For example, companies would use advertising as a propaganda tool to inform or influence customers to buy and provide good evaluations for the products. During the process, the customers' opinions or preferences may change dynamically, which has a great impact on markets. It is important to choose appropriate decision-making and publicity methods to discover the preferences of customers by mining data that emerge online and offline and to influence the opinions and behavior of consumers in order to achieve the desired effect. Particularly, related methods need validation in real case studies.

3) Complex linguistic decision-making: The development of information technology enables people to share their reviews about products and events online. In most cases, online reviews are presented using linguistic information under complex contexts, such as dynamic social networks, heterogeneous decision environments and large-scale decision environments. In addition, in linguistic decision-making, the fact that words mean different things for different people indicates the necessity of personalizing individual semantics on the representation of CW. As methodologies for exploring online reviews have been widely studied, they need validation in practical complex linguistic decision-making environments based on personalized individual semantics[110].

\section{CONCLUDing REMARKS}

There exists a prolific literature on fuzzy decision-making as a result of studies made along the last years, together with a wealth of models. They have contributed to make the area of fuzzy sets and CW-based decision-making one of the most prolific areas in the field of fuzzy set theory.

Taking a critical glance at this prolific journey, probably and certainly, many fuzzy decision-making models can be far from reality in the representations of the assessments. Or (also certainly) the mathematical decision models themselves can 
be too complex for a problem that should be closer to human common sense, that is, in the representation of uncertainty and in the process of human reasoning in decision-making. We must rethink, revisit, and redirect studies, trying to: be more realistic, avoid the possibility of saying that publications are in many cases being produced under the pressing necessity of "publish or perish", and adopt a more critical view in many areas where extensions of fuzzy models emerge to represent uncertainty but they end up being loaded with much greater uncertainty for their use. Moreover, we stress the pressing need for performance evaluation and real-world validation standards that guarantee a real (beyond purely theoretical) value in present and future contributions in the area.

Conversely, on a more positive glance, we are facing interesting discussions for future research in this area, with interesting possibilities that open as our society becomes increasingly digital, with social networks guiding society opinions, and where data (for driving decisions) is "the current oil of the XXI century". Now, at the beginning of a new decade where Artificial Intelligence becomes the driving force of digital transformation, data will lead to new fuzzy decision models based on personalized recommendations, assessments extracted from opinions beneath data, etc., thereby leading us to an era of smarter decision aid tools.

In short, new scenarios will be proposed where uncertainty and common sense have to be a key part of the whole situation, in decision models that will include the wisdom of crowds decision-making. Artificial Intelligence combined with decision theory will make it possible, to design models that form wiser decisions in better ways.

With this paper we hope to have contributed a little to rethinking the current state of affairs in the field of fuzzy decision-making, rethinking the problems and applying common sense to decision-making, in representations, models, and solutions to manage uncertainty inherent in all decisionmaking processes. Reiterating on the "common sense" and remembering with it our scientific father and dear Lofti Zadeh, with his paper [55]: "Common sense knowledge representation based on fuzzy logic".

\section{ACKNOWLEDGMENT}

The authors would like to thank the support provided by the Spanish State Research Agency through the projects PID2019103880RB-I00 / AEI / 10.13039/501100011033 and TIN201675850-R, the NSF of China through the project 71901182, and the China Postdoctoral Science Foundation through the project 2020 M673283.

\section{REFERENCES}

[1] L. A. Zadeh, "Fuzzy sets," Information and Control, vol. 5, no. 3, pp. 338-353, 1965.

[2] R. E. Bellman and L. A. Zadeh, "Decision making in a fuzzy environment," in Management Science, vol. 17, no. 4, 1970, pp. 141-164.

[3] M. Zeleny, Multiple Criteria Decision Making. McGraw-Hill, 1982.

[4] M. G. Shendrik and G. B. Tamm, "An approach to interactive solution of multicriterial optimization problems with linquistic modeling of preferences," Automatic Control and Computer Sciences, vol. 19, no. 6, pp. 1-7, 1986.

[5] J. Kacprzyk, "Group decision making with a fuzzy linguistic majority," Fuzzy Sets and Systems, vol. 18, no. 2, pp. 105-118, 1986.
[6] R. M. Tong and P. P. Bonissone, "A linguistic approach to decisionmaking with fuzzy sets," IEEE Transactions on Systems, Man, and Cybernetics, vol. 10, no. 11, pp. 716-723, 1980.

[7] R. R. Yager, "A new methodology for ordinal multiobjective decisions based on fuzzy sets," Decision Sciences, vol. 12, no. 4, pp. 589-600, 1981.

[8] L. A. Zadeh, "Fuzzy logic = computing with words," IEEE Transactions on Fuzzy Systems, vol. 4, no. 2, pp. 103-111, 1996.

[9] J. Kacprzyk, M. Fedrizzi, and H. Nurmi, "Group decision making and consensus under fuzzy preferences and fuzzy majority," Fuzzy Sets and Systems, vol. 49, no. 1, pp. 21-31, 1992.

[10] F. Herrera and L. Martínez, "A 2-tuple fuzzy linguistic representation model for computing with words," IEEE Transactions on Fuzzy Systems, vol. 8, no. 6, pp. 746-752, 2000.

[11] X. Gou, H. Liao, Z. S. Xu, and F. Herrera, "Double hierarchy hesitant fuzzy linguistic term set and MULTIMOORA method: A case of study to evaluate the implementation status of haze controlling measures," Information Fusion, vol. 38, pp. 22-34, 2017.

[12] R. R. Yager, "On ordered weighted averaging aggregation operators in multicriteria decisionmaking," IEEE Transactions on Systems, Man, and Cybernetics, vol. 18, no. 1, pp. 183-190, 1988.

[13] Z. S. Xu and N. Zhao, "Information fusion for intuitionistic fuzzy decision making: An overview," Information Fusion, vol. 28, pp. 1023, 2016.

[14] J. Wu and F. Chiclana, "A social network analysis trust-consensus based approach to group decision-making problems with intervalvalued fuzzy reciprocal preference relations," Knowledge-Based Systems, vol. 59, pp. 91-107, 2014.

[15] Y. C. Dong, Q. Zha, H. Zhang, G. Kou, H. Fujita, F. Chiclana, and E. Herrera-Viedma, "Consensus reaching in social network group decision making: Research paradigms and challenges," KnowledgeBased Systems, vol. 162, pp. 3-13, 2018.

[16] I. Palomares, L. Martínez, and F. Herrera, "A consensus model to detect and manage noncooperative behaviors in large-scale group decision making," IEEE Transactions on Fuzzy Systems, vol. 22, no. 3, pp. 516530, 2014.

[17] C. Zuheros, E. Martínez-Cámara, E. Herrera-Viedma, and F. Herrera, "Sentiment analysis based multi-person multi-criteria decision making methodology using natural language processing and deep learning for smarter decision aid. Case study of restaurant choice using TripAdvisor reviews," Information Fusion, vol. 68, pp. 22-36, 2021.

[18] H. Bustince, E. Barrenechea, M. Pagola, J. Fernández, Z. S. Xu, B. Bedregal, J. Montero, H. Hagras, F. Herrera, and B. De Baets, "A historial account of types of fuzzy sets and their relationships," IEEE Transactions on Fuzzy Systems, vol. 24, no. 1, pp. 179-194, 2016.

[19] J. G. Dijkman, H. van Haeringen, and S. J. de Lange, "Fuzzy numbers," Journal of Mathematical Analysis and Applications, vol. 92, no. 2, pp. 301-341, 1983.

[20] P. J. M. van Laarhoven and W. Pedrycz, "A fuzzy extension of saaty's priority theory," Fuzzy Sets and Systems, vol. 11, no. 1-3, pp. 229-241, 1983.

[21] T. Bilgiç, "Interval-valued preference structures," European Journal of Operational Research, vol. 105, no. 1, pp. 162-183, 1998.

[22] K. T. Atanassov, "Intuitionistic fuzzy sets," Fuzzy Sets and Systems, vol. 20 , no. 1 , pp. 87-96, 1986

[23] H. Liao, X. Mi, Z. S. Xu, J. Xu, and F. Herrera, "Intuitionistic fuzzy analytic network process," IEEE Transactions on Fuzzy Systems, vol. 26, no. 5, pp. 2578-2590, 2018.

[24] E. Szmidt and J. Kacprzyk, "A consensus-reaching process under intuitionistic fuzzy preference relations," International Journal of Intelligent Systems, vol. 18, no. 7, pp. 837-852, 2003.

[25] G. Deschrijver and E. E. Kerre, "On the relationship between some extensions of fuzzy set theory," Fuzzy Sets and Systems, vol. 133, no. 2 , pp. 227-235, 2003.

[26] V. Torra, "Hesitant fuzzy sets," International Journal of Intelligent Systems, vol. 25, no. 6, pp. 529-539, 2010.

[27] R. M. Rodríguez, B. Bedregal, H. Bustince, Y. C. Dong, B. Farhadinia, C. Kahraman, L. Martínez, V. Torra, Y. J. Xu, Z. S. Xu, and F. Herrera, "A position and perspective analysis of hesitant fuzzy sets on information fusion in decision making. Towards high quality progress," Information Fusion, vol. 29, pp. 89-97, 2016.

[28] R. M. Rodríguez, L. Martínez, V. Torra, Z. S. Xu, and F. Herrera, "Hesitant fuzzy sets: State of the art and future directions," International Journal of Intelligent Systems, vol. 29, no. 6, pp. 495-524, 2014.

[29] F. Herrera, S. Alonso, F. Chiclana, and E. Herrera-Viedma, "Computing with words in decision making: Foundations, trends and prospects," 
Fuzzy Optimization and Decision Making, vol. 8, no. 4, pp. 337-364, 2009.

[30] L. A. Zadeh, "The concept of a linguistic variable and its application to approximate reasoning-I," Information Sciences, vol. 8, no. 3, pp. 199-249, 1975.

[31] _ - "The concept of a linguistic variable and its application to approximate reasoning-II," Information Sciences, vol. 8, no. 4, pp. 301-357, 1975.

[32] — " "The concept of a linguistic variable and its application to approximate reasoning-III," Information Sciences, vol. 9, no. 1, pp. $43-80,1975$.

[33] C. Zuheros, C.-C. Li, F. J. Cabrerizo, Y. C. Dong, E. Herrera-Viedma, and F. Herrera, "Computing with words: Revisiting the qualitative scale," International Journal of Uncertainty, Fuzziness and KnowledgeBased Systems, vol. 26, no. Suppl. 2, pp. 127-143, 2018.

[34] R. Degani and G. Bortolan, "The problem of linguistic approximation in clinical decision making," FInternational Journal of Approximate Reasoning, vol. 2, no. 2, pp. 143-162, 1988.

[35] F. Liu and J. M. Mendel, "Encoding words into interval type-2 fuzzy sets using an interval approach," IEEE Transactions on Fuzzy Systems, vol. 16, no. 6, pp. 1503-1521, 2008.

[36] M. Delgado, J. L. Verdeay, and M. A. Vila, "On aggregation operations of linguistic labels," International Journal of Intelligent Systems, vol. 8, no. 3, pp. 351-370, 1993.

[37] Z. S. Xu, "A method based on linguistic aggregation operators for group decision making with linguistic preference relations," Information Sciences, vol. 166, no. 1-4, pp. 19-30, 2004.

[38] Z. S. Xu and H. Wang, "On the syntax and semantics of virtual linguistic terms for information fusion in decision making," Information Fusion, vol. 34, pp. 43-48, 2017.

[39] J. L. García-Lapresta and D. Pérez-Román, “Ordinal proximity measures in the context of unbalanced qualitative scales and some applications to consensus and clustering," Applied Soft Computing, vol. 35 , pp. 864-872, 2015.

[40] F. Herrera and L. Martínez, "A model based on linguistic 2-tuples for dealing with multigranular hierarchical linguistic contexts in multiexpert decision making," IEEE Transactions on Systems, Man, and Cybernetics, Part B, vol. 31, no. 2, pp. 227-234, 2001

[41] F. Herrera, E. Herrera-Viedma, and L. Martínez, "A fuzzy linguistic methodology to deal with unbalanced linguistic term sets," IEEE Transactions on Fuzzy Systems, vol. 16, no. 2, pp. 354-370, 2008

[42] J. Wang and J. Hao, "A new version of 2-tuple fuzzy linguistic representation model for computing with words," IEEE Transactions on Fuzzy Systems, vol. 14, no. 3, pp. 435-445, 2006.

[43] Y. C. Dong, Y. Xu, and S. Yu, "Computing the numerical scale of the linguistic term set for the 2-tuple fuzzy linguistic representation model," IEEE Transactions on Fuzzy Systems, vol. 17, no. 6, pp. 1366-1378, 2009.

[44] H. Liao, R. Qin, C. Gao, X. Wu, A. Hafezalkotob, and F. Herrera, "Score-HeDLiSF: A score function of hesitant fuzzy linguistic term set based on hesitant degrees and linguistic scale functions: An application to unbalanced hesitant fuzzy linguistic MULTIMOORA," Information Fusion, vol. 48, pp. 39-54, 2019.

[45] R. M. Rodríguez, A. Labella, and L. Martínez, "An overview on fuzzy modelling of complex linguistic preferences in decision making," Intertional Journal of Computational Intelligence Systems, vol. 9, no. Sup. 1, pp. 81-94, 2016.

[46] H. Wang, Z. S. Xu, and X.-J. Zeng, "Modeling complex linguistic expressions in qualitative decision making: An overview," KnowledgeBased Systems, vol. 144, pp. 174-187, 2018.

[47] Y. Wu, Z. Zhang, G. Kou, H. Zhang, X. Chao, C.-C. Li, Y. C. Dong, and F. Herrera, "Distributed linguistic representations in decision making: Taxonomy, key elements and applications, and challenges in data science and explainable artificial intelligence," Information Fusion, vol. 65 , pp. 165-178, 2021.

[48] R. M. Rodríguez, L. Martínez, and F. Herrera, "Hesitant fuzzy linguistic term sets for decision making," IEEE Transactions on Fuzzy Systems, vol. 20, no. 1, pp. 109-119, 2012.

[49] G. Q. Zhang, Y. C. Dong, and Y. F. Xu, "Consistency and consensus measures for linguistic preference relations based on distribution assessments," Information Fusion, vol. 17, pp. 46-55, 2014.

[50] R. R. Yager, "Pythagorean fuzzy subsets," in Proceedings of the 2013 Joint IFSA World Congress and NAFIPS Annual Meeting (IFSA/NAFIPS), Edmonton, Canada, June 2013, pp. 57-61.

[51] B. C. Cuong and V. Kreinovich, "Picture fuzzy sets - A new concept for computational intelligence problems," in Proceedings of the 2013 Third
World Congress on Information and Communication Technologies (WICT 2013), Hanoi, Vietnam, December 2013, pp. 1-6.

[52] R. R. Yager, "Generalized orthopair fuzzy sets," IEEE Transactions on Fuzzy Sets, vol. 25, no. 5, pp. 1222-1230, 2017.

[53] Z. Hao, Z. S. Xu, H. Zhao, and Z. Su, "Probabilistic dual hesitant fuzzy set and its application in risk evaluation," Knowledge-Based Systems, vol. 127, pp. 16-28, 2017.

[54] T. Senapati and R. R. Yager, "Fermatean fuzzy sets," Journal of Ambient Intelligence and Humanized Computing, vol. 11, pp. 663-674, 2020.

[55] L. A. Zadeh, "Commonsense knowledge representation based on fuzzy logic," Computer, vol. 16, no. 10, pp. 61-65, 1983.

[56] B. Roy, "Paradigms and challenges," in Multiple Criteria Decision Analysis: State of the Art Surveys, 2nd ed., S. Greco, M. Ehrgott, and J. R. Figueira, Eds. New York: Springer-Verlag, 2016, pp. 19-39.

[57] S. Greco, M. Ehrgott, and J. R. F. (Eds.), Multiple Criteria Decision Analysis: State of the Art Surveys. New York: Springer-Verlag, 2016.

[58] B. Ceballos, M. T. Lamata, and D. Pelta, "A comparative analysis of multi-criteria decision making methods," Progress in Artificial Intelligence, vol. 5, pp. 315-322, 2016.

[59] P. Wang, Z. Zhu, and Y.-H. Wang, "A novel hybrid MCDM model combining the SAW, TOPSIS and GRA methods based on experimental design," Information Sciences, vol. 345, pp. 27-45, 2016.

[60] G. Stojić, Z. Stević, J. Antuchevičienè, D. Pamučar, and M. Vasiljević, "A novel rough WASPAS approach for supplier selection in a company manufacturing PVC carpentry products," Information, vol. 9, no. 5, p. 121,2018

[61] C.-L. Hwang and K. Yoon, Multiple Attribute Decision Making: Methods and Applications - A State-of-the-Art Survey. Berlin, Heidelberg: Springer-Verlag, 1981.

[62] S. Opricovic and G.-H. Tzeng, "Compromise solution by MCDM methods: A comparative analysis of VIKOR and TOPSIS," European Journal of Operational Research, vol. 156, no. 2, pp. 445-455, 2004.

[63] J. P. Brans, P. Vincke, and B. Mareschal, "How to select and how to rank projects: The PROMETHEE method," European Journal of Operational Research, vol. 24, no. 2, pp. 228-238, 1986.

[64] M. Roubens, "Preference relations on actions and criteria in multicriteria decision making," European Journal of Operational Research, vol. 10, no. 1, pp. 51-55, 1982.

[65] K. Govindan and M. B. Jepsen, "ELECTRE: A comprehensive literature review on methodologies and applications," European Journal of Operational Research, vol. 250, no. 1, pp. 1-29, 2016.

[66] K. Sekitani and I. Takahashi, "A unified model and analysis for AHP and ANP," Journal of The Operations Research Society of Japan, vol. 44, no. 1, pp. 67-89, 2001.

[67] A. Hafezalkotob, H. Liao, and F. Herrera, "An overview of MULTIMOORA for multi-criteria decision making: Theory, developments, applications, and challenges," Information Fusion, vol. 51, pp. 145177, 2019.

[68] L. Muñoz Moro and A. Ramos, "Goal programming approach to maintenance scheduling of generating units in large scale power systems," IEEE Transactions on Power Systems, vol. 14, no. 3, pp. 1021-1028, 1999.

[69] R. Pelissari, M. A. Oliveira, A. J. Abackerli, S. Ben-Amor, and M. R. P. Assumpção, "Techniques to model uncertain input data of multicriteria decision making problems: A literature review," International Transactions in Operational Research, vol. 28, no. 2, pp. 523-559, 2021.

[70] Z. Chen, K. Chin, Y. Li, and Y. Yang, "Proportional hesitant fuzzy linguistic term set for multiple criteria group decision making," Inf. Sci., vol. 357, pp. 61-87, 2016

[71] R. L. Keeney and R. S. Gregory, "Selecting attributes to measure the achievement of objectives," Opererations Research, vol. 53, no. 1, pp. $1-11,2005$.

[72] H. Doukas, A. Tsiousi, V. Marinakis, and J. Psarras, "Linguistic multi-criteria decision making for energy and environmental corporate policy," Information Sciences, vol. 258, pp. 328-338, 2014.

[73] W. Pedrycz, P. Ekel, and R. Parreiras, Fuzzy Multicriteria Decision Making: Models, Methods and Applications. John Wiley \& Son, 2010.

[74] G. Beliakov, A. Pradera, and T. Calvo, Aggregation Functions: A Guide for Practitioners. Berlin, Heidelberg: Springer-Verlag, 2007.

[75] M. Grabisch and M. Roubens, "Application of the Choquet integral in multicriteria decision making," in Fuzzy Measures and Integrals. Theory and Applications, M. Grabisch, T. Murofushi, and M. Sugeno, Eds. Heidelberg: Physica-Verlag, 2000, pp. 348-374. 
[76] R. R. Yager, "The power average operator," IEEE Transactions on Systems, Man, and Cybernetics, Part A: Systems and Humans, vol. 31, no. 6, pp. 724-731, 2001.

[77] I. Kaya, M. Çolak, and F. Terzi, "A comprehensive review of fuzzy multi criteria decision making methodologies for energy policy making," Energy Strategy Reviews, vol. 24, pp. 207-228, 2019.

[78] H.-W. Liu and G.-J. Wang, "Multi-criteria decision making methods based on intuitionistic fuzzy sets," European Journal of Operational Research, vol. 179, no. 1, pp. 220-233, 2007.

[79] S. Nădăban, S. Dzitac, and I. Dzitac, "Fuzzy topsis: A general view," Procedia Computer Science, vol. 91, pp. 823-831, 2016.

[80] H. Liao, Z. S. Xu, and X.-J. Zeng, "Hesitant fuzzy linguistic VIKOR method and its application in qualitative multiple criteria decision making," IEEE Transactions on Fuzzy Systems, vol. 23, no. 5, pp. 1343-1355, 2015.

[81] J. Li, Q. Chen, L.-L. Niu, and Z.-X. Wang, "An ORESTE approach for multi-criteria decision-making with probabilistic hesitant fuzzy information," International Journal of Machine Learning and Cybernetics, vol. 11, pp. 1591-1609, 2020

[82] W. Li, S. Yu, H. Pei, C. Zhao, and B. Tian, "A hybrid approach based on fuzzy AHP and 2-tuple fuzzy linguistic method for evaluation inflight service quality," Journal of Air Transport Management, vol. 60, pp. 49-64, 2017.

[83] S. Moradpour and S. Long, "Using combined multi-criteria decision making and data mining methods for work zone safety: A case analysis," Case Studies on Transport Policy, vol. 7, no. 2, pp. 178184, 2019.

[84] F. Herrera, E. Herrera-Viedma, and J. L. Verdegay, "Direct approach processes in group decision making using linguistic OWA operators," Fuzzy Sets and Systems, vol. 79, no. 2, pp. 175-190, 1996.

[85] I. Palomares, Large Group Decision Making: Creating Decision Support Approaches at Scale. Springer, 2018

[86] S. A. Orlovsky, "Decision-making with a fuzzy preference relation," Fuzzy Sets and Systems, vol. 1, no. 3, pp. 155-167, 1978.

[87] M. Roubens, "Fuzzy sets and decision analysis," Fuzzy Sets and Systems, vol. 90, no. 2, pp. 199-206, 1997.

[88] E. Herrera-Viedma, F. J. Cabrerizo, J. Kacprzyk, and W. Pedrycz, "A review of soft consensus models in a fuzzy environment," Information Fusion, vol. 17, pp. 4-13, 2014.

[89] Y. C. Dong and J. Xu, Consensus Building in Group Decision Making. Singapore: Springer, 2016

[90] I. Palomares, F. J. Estrella, L. Martínez, and F. Herrera, "Consensus under a fuzzy context: Taxonomy, analysis framework AFRYCA and experimental case of study," Information Fusion, vol. 20, pp. 252-271, 2014.

[91] H. Zhang, S. Zhao, G. Kou, C.-C. Li, Y. C. Dong, and F. Herrera, "An overview on feedback mechanisms with minimum adjustment or cost in consensus reaching in group decision making: Research paradigms and challenges," Information Fusion, vol. 60, pp. 65-79, 2020.

[92] M. J. del Moral, F. Chiclana, J. M. Tapia, and E. Herrera-Viedma, "A comparative study on consensus measures in group decision making," International Journal of Intelligent Systems, vol. 33, no. 8, pp. 1624$1638,2018$.

[93] F. J. Cabrerizo, R. Ureña, W. Pedrycz, and E. Herrera-Viedma, "Building consensus in group decision making with an allocation of information granularity," Fuzzy Sets and Systems, vol. 255, pp. 115127, 2014.

[94] S. Saint and J. Lawson, Rules for Reaching Consensus: A Modern Approach to Decision Making. John Wiley \& Sons, 1994.

[95] M. Cao, J. Wu, F. Chiclana, R. Ureña, and E. Herrera-Viedma, "A personalized consensus feedback mechanism based on maximum harmony degree," IEEE Transactions on Systems, Man, and Cybernetics: Systems, pp. 1-13, in press.

[96] Z. Gong, X. Xu, W. Guo, E. Herrera-Viedma, and F. J. Cabrerizo, "Minimum cost consensus modelling under various linear uncertainconstrained scenarios," Information Fusion, vol. 66, pp. 81-17, 2021.

[97] J. Wu, L. Dai, F. Chiclana, H. Fujita, and E. Herrera-Viedma, "A minimum adjustment cost feedback mechanism based consensus model for group decision making under social network with distributed linguistic trust," Information Fusion, vol. 41, pp. 232-242, 2017.

[98] I. J. Pérez, F. J. Cabrerizo, S. Alonso, and E. Herrera-Viedma, "A new consensus model for group decision making problems with nonhomogeneous experts," IEEE Transactions on Systems, Man, and Cybernetics: Systems, vol. 44, no. 4, pp. 494-498, 2014.

[99] Z. Wu and J. Xu, "Managing consistency and consensus in group decision making with hesitant fuzzy linguistic preference relations,"
Omega-international Journal of Management Science, vol. 65, pp. 28 40, 2016

[100] X. Chen, H. Zhang, and Y. C. Dong, "The fusion process with heterogeneous preference structures in group decision making: A survey," Information Fusion, vol. 24, pp. 72-83, 2015.

[101] G. Li, G. Kou, and Y. Peng, "A group decision making model for integrating heterogeneous information," IEEE Transactions on Systems, Man, and Cybernetics: Systems, vol. 48, no. 6, pp. 982-992, 2018.

[102] H.-B. Yan and T. Ma, "A group decision making approach to uncertain quality function deployment based on fuzzy preference relation and fuzzy majority," European Journal of Operational Research, vol. 241, no. 3, pp. 815-829, 2015.

[103] D. Cheng, F. Cheng, Z. Zhou, and Y. Wu, "Reaching a minimum adjustment consensus in social network group decision making," Information Fusion, vol. 59, pp. 30-43, 2020.

[104] H. Zhang, I. Palomares, Y. C. Dong, and W. Wang, "Managing non-cooperative behaviors in consensus-based multiple attribute group decision making: An approach based on social network analysis," Knowledge-Based Systems, vol. 162, pp. 29-45, 2018.

[105] R.-X. Ding, I. Palomares, X. Wang, G.-R. Yang, B. Liu, Y. C. Dong, E. Herrera-Viedma, and F. Herrera, "Large-scale decision making: Characterization, taxonomy, challenges and future directions from an Artificial Intelligence and applications perspective," Information Fusion, vol. 59, pp. 84-102, 2020.

[106] R.-X. Ding, X. Wang, K. Shang, B. Liu, and F. Herrera, "Sparse representation-based intuitionistic fuzzy clustering approach to find the group intra-relations and group leaders for large-scale decision making," IEEE Transactions on Fuzzy Systems, vol. 27, no. 3, pp. 559573, 2019.

[107] Y. Liu, Y. C. Dong, H. Liang, F. Chiclana, and E. Herrera-Viedma, "Multiple attribute strategic weight manipulation with minimum cost in a group decision making context with interval attribute weights information," IEEE Transactions on Systems, Man, and Cybernetics: Systems, vol. 49, no. 10, pp. 1981-1992, 2019.

[108] Y. C. Dong, M. Zhan, G. Kou, Z. Ding, and H. Liang, "A survey on the fusion process in opinion dynamics," Information Fusion, vol. 43 , pp. 57-65, 2018.

[109] B. Liu, Q. Zhou, R.-X. Ding, I. Palomares, and F. Herrera, "Largescale group decision making model based on social network analysis: Trust relationship-based conflict detection and elimination," European Journal of Operational Research, vol. 275, no. 2, pp. 737-754, 2019.

[110] C.-C. Li, Y. C. Dong, F. Herrera, E. Herrera-Viedma, and L. Martínez, "Personalized individual semantics in computing with words for supporting linguistic group decision making. An application on consensus reaching," Information Fusion, vol. 33, pp. 29-40, 2017.

[111] R. R. Yager, "Non-numeric multi-criteria multi-person decision making," Group Decision and Negotiation, vol. 2, pp. 81-93, 1993.

[112] K. T. Atanassov, G. Pasi, and R. R. Yager, "Intuitionistic fuzzy interpretations of multi-person multi-criteria decision making," in Proceedings First International IEEE Symposium Intelligent Systems, Varna, Bulgaria, September 2002, pp. 115-119.

[113] T.-C. Wen, H.-H. Lai, and K.-H. Chang, "A new flexible method for solving multi-expert multi-criterion decision making problems," Applied Sciences, vol. 10, no. 13, p. 4582, 2020.

[114] J. Gao, F. Guo, Z. Ma, X. Huang, and X. Li, "Multi-criteria group decision making framework for offshore wind farm site selection based on the intuitionistic linguistic aggregation operators," Energy, vol. 204, p. 117899, 2020.

[115] J. Wang, J.-Q. Wang, and Y.-X. Ma, "Possibility degree and power aggregation operators of single-valued trapezoidal neutrosophic numbers and applications to multi-criteria group decision making," Cognitive Computation, pp. 1-16, 2020

[116] H. Sellak, B. Ouhbi, B. Frikh, and B. Ikken, "Expertise-based consensus building for MCGDM with hesitant fuzzy linguistic information," Information Fusion, vol. 50, pp. 54-70, 2019.

[117] I. Palomares, M. Crosscombe, Z.-S. Chen, and J. Lawry, "Dual consensus measure for multi-perspective multi-criteria group decision making," in Proceedings of the 2018 IEEE International Conference on Systems, Man, and Cybernetics (SMC), Miyazaki, Japan, October 2018, pp. 3313-3318.

[118] P. Liu and Y. Li, "An extended MULTIMOORA method for probabilistic linguistic multi-criteria group decision making based on prospect theory," Computers \& Industrial Engineering, vol. 136, pp. 528-545, 2019.

[119] E. Herrera-Viedma, F. J. Cabrerizo, F. Chiclana, J. Wu, M. J. Cobo, and K. Samuylov, "Consensus in group decision making and social 
networks," Studies in Informatics and Control, vol. 26, no. 3, pp. 259268, 2017.

[120] I. J. Pérez, P. García-Sánchez, F. J. Cabrerizo, and E. Herrera-Viedma "An approach toward a feedback mechanism for consensus reaching processes using gamification to increase the experts' experience," in Proceedings of the 53rd Hawaii International Conference on System Sciences (HICSS 53), Maui, Hawaii, USA, January 2020, pp. $1717-$ 1726.

[121] R. Hegselmann and U. Krause, "Opinion dynamics and bounded confidence models, analysis and simulation," Journal of Artificial Societies \& Social Simulation, vol. 5, no. 3, pp. 2-33, 2002.

[122] Z. Zheng, S. Xie, H.-N. Dai, X. Chen, and H. Wang, "Blockchain challenges and opportunities: A survey," International Journal of Web and Grid Services, vol. 14, no. 4, pp. 352-375, 2018.

[123] W. D. Eggers, J. Guzscza, and M. Greene, "Making cities smarter: How citizens' collective intelligence can guide better decision making," Deloitte Review, Issue 20, 2017.

[124] C. C. Aggarwal, Recommender Systems: The Textbook. Cham: Springer International Publishing, 2016.

[125] A. Felfernig, L. Boratto, M. Stettinger, and M. Tkalčič, Group Recommender Systems: An Introduction. Springer, 2018

[126] D. Monti, G. Rizzo, and M. Morisio, "A systematic literature review of multicriteria recommender systems," Artificial Intelligence Review, in press.

[127] J. Liu, M. Kadzinski, X. Liao, and X. Mao, "Data-driven preference learning methods for value-driven multiple criteria sorting with interacting criteria," INFORMS Journal on Computing, in press. DOI: 10.1287/ijoc.2020.0977.

[128] R. Ureña, F. J. Cabrerizo, J. A. Morente-Molinera, and E. HerreraViedma, "GDM-R: A new framework in $\mathrm{r}$ to support fuzzy group decision making processes," Inf. Sci., vol. 357, pp. 161-181, 2016.

[129] D. Molina, A. LaTorre, and F. Herrera, "An insight into bio-inspired and evolutionary algorithms for global optimization: Review, analysis, and lessons learnt over a decade of competitions," Cognitive Computation, vol. 10, pp. 517-544, 2018.

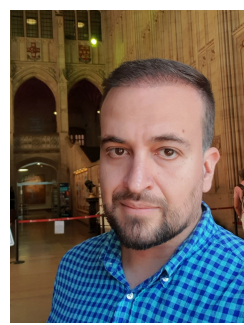

Iván Palomares received two M.Sc. degrees, one in computer science (with Faculty and Nationwide Distinctions) from the University of Jaén, Spain; and one in Soft Computing and Intelligent Systems (with Honors) from the University of Granada, Spain, in 2009 and 2011, respectively. He received the $\mathrm{Ph} . \mathrm{D}$. degree in computer science (with Nationwide distinctions) from the University of Jaén, in 2014. $\mathrm{He}$ is a Senior Research Scientist at the Andalusian Research Institute in Data Science and Artificial Intelligence (DaSCI), University of Granada, Spain, and honorary Lecturer in Data Science and Artificial Intelligence with the University of Bristol, UK. He has been a Turing Fellow with the Alan Turing Institute, London, UK, between 2018-2020. He has authored a book "Large Group Decision Making: Creating Decision Support Systems at Scale." His research interests include data-driven and intelligent approaches for recommender systems, personalization for health and wellbeing, leisure and tourism in smart cities, reciprocal recommender systems for social matching, large-scale decision making and consensus, preference modelling and aggregation, and AI for Sustainable Development Goals. Dr. Palomares's research results have been published in top journals and conference proceedings, including IEEE TRANSACTIONS ON FUZZY SYSTEMS; European Journal of Operational Research; Applied Soft Computing; International Journal of Intelligent Systems; Information Fusion, Knowledge-Based Systems; Data and Knowledge Engineering; Renewable \& Sustainable Energy Reviews, amongst others.

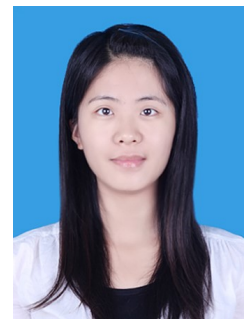

Cong-Cong $\mathrm{Li}$ received the $\mathrm{Ph} . \mathrm{D}$. degree in management from Sichuan University, China; and the Ph.D. degree in computer science and artificial intelligence from university of Granada, Spain, both in 2018. Currently, she is a Lecturer with the School of Economics and Management, Southwest Jiaotong University, Chengdu, China.

Her research interests include decision making, computing with words and opinion dynamics. Her research results have been published in some refereed journals, including IEEE Transactions on Fuzzy Systems, IEEE Transactions on SMC: Systems, Information Fusion, Information Sciences, and Group Decision and Negotiation, among others.

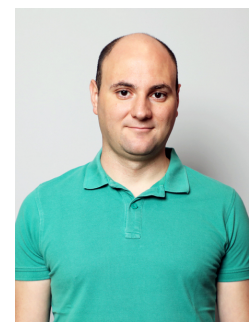

Francisco Javier Cabrerizo was born in Jódar, Jaén, Spain, in 1983. He received the M.S. and Ph.D. degrees in computer science from the University of Granada, Spain, in 2006 and 2008, respectively.

From 2008 to 2016, he was an Assistant Professor with the Department of Software Engineering and Computer Systems, National Distance Education University (UNED), Madrid, Spain. Since 2016, he has been an Associate Professor with the Department of Computer Science and Artificial Intelligence, University of Granada, Spain. He is an Associate Editor of IEEE Transactions on Cybernetics and Journal of Intelligent \& Fuzzy Systems. He has published extensively in leading international journals, and several of his papers (currently 14) are classified as Highly Cited Papers in Essential Science Indicators from Clarivate Analytics. His h-index is 28 and he presents more than 4100 citations in Web of Science from Clarivate Analytics. He has been identified as Highly Cited Researcher by Clarivate Analytics in 2018, 2019 and 2020. His research interest includes fuzzy decision making, decision support systems, consensus models, linguistic modeling, aggregation of information, digital libraries, web quality evaluation, and bibliometrics.

Dr. Cabrerizo's awards and honors include the ISDA 2009 Conference Best Paper Award, the ICSEE 2015 Conference Best Paper Award, the 2014 Herbert Simon Award for outstanding contribution in International Journal of Information Technology \& Decision Making, and the 2016 Andrew P. Sage Best Transactions Paper Award, which recognizes the authors of the best paper published annually in IEEE Transactions on Systems, Man, and Cybernetics: Systems. 


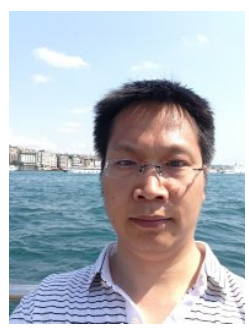

Yucheng Dong received the B.S. and M.S. degrees in mathematics from Chongqing University, Chongqing, China, in 2002 and 2004, respectively, and the Ph.D. degree in management from Xi'an Jiaotong University, Xi'an, China, in 2008. He is currently a Professor with the Business School, Sichuan University, Chengdu, China. His current research interests include decision analysis, human dynamics, big data analytics, social network, and risk analysis. He has authored or coauthored more than 100 international journal papers in Decision Support Systems, European Journal of Operational Research, IEEE Transactions on big data, IEEE Transactions on Cybernetic, IEEE Transactions on fuzzy systems, IEEE Transactions on computational social systems, IEEE Transactions on SMC: Systems, IEEE Transactions on Reliability, Omega, and Scientific Data. He has been identified by Clarivate as Highly Cited Researcher in the field of computer science. Dr. Dong is an Area Editor/Associate Editor of Computers and Industrial Engineering, Group Decision and Negotiation, the IEEE TRANSACTIONS ON SMC: SYSTEMS, and Information Fusion.

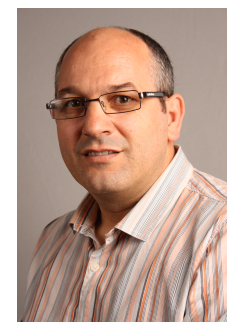

Francisco Chiclana received the $\mathrm{BSc}$ and $\mathrm{PhD}$ in Mathematics from the University of Granada, Spain, in 1989 and 2000, respectively. He is a Professor of Computational Intelligence and Decision Making with the School of Computer Science and Informatics, Faculty of Computing, Engineering and Media, De Montfort University, Leicester, U.K. He is an Associate Editor and a Guest Editor for several ISI indexed journals. He has organized and chaired special sessions/workshops in many major international conferences in research areas as fuzzy preference modeling, decision support systems, consensus, recommender systems, social networks, rationality/consistency, aggregation. He is currently a Highly Cited Researcher in Computer Sciences (according to Essential Science Indicators by Clarivate Analytics).

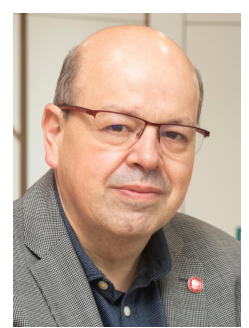

Francisco Herrera (SM'15) received his M.Sc. in Mathematics in 1988 and Ph.D. in Mathematics in 1991, both from the University of Granada, Spain. $\mathrm{He}$ is a Professor in the Department of Computer Science and Artificial Intelligence at the University of Granada and Director of the Andalusian Research Institute in Data Science and Computational Intelligence (DaSCI). He's an academician in the Royal Academy of Engineering (Spain).

He has been the supervisor of 51 Ph.D. students. He has published more than 500 journal papers, receiving more than 90000 citations (Scholar Google, H-index 147). He has been nominated as a Highly Cited Researcher (in the fields of Computer Science and Engineering, respectively, 2014 to present, Clarivate Analytics). $\mathrm{He}$ currently acts as Editor in Chief of the international journal "Information Fusion" (Elsevier). He acts as editorial member of a dozen of journals.

He received the several honors and awards, among others: ECCAI Fellow 2009, IFSA Fellow 2013, 2010 Spanish National Award on Computer Science ARITMEL to the "Spanish Engineer on Computer Science", International Cajastur "Mamdani" Prize for Soft Computing (Fourth Edition, 2010), IEEE Transactions on Fuzzy System Outstanding 2008 and 2012 Papers, 2011 Lotfi A. Zadeh Prize Best paper Award (IFSA Association), 2013 AEPIA Award to a scientific career in Artificial Intelligence, 2014 XV Andalucía Research Prize Maimónides, 2017 Andalucía Medal (by the regional government of Andalucía), 2018 "Granada: Science and Innovation City" award.

His current research interests include among others, Computational Intelligence (including fuzzy modeling, computing with words, evolutionary algorithms and deep learning), information fusion and decision making, and data science (including data preprocessing, prediction, non-standard classification problems, and big data). 